\title{
The AMBRE project: Constraining the lithium evolution in the Milky Way*
}

\author{
G. Guiglion ${ }^{1}$, P. de Laverny ${ }^{1}$, A. Recio-Blanco ${ }^{1}$, C. C. Worley ${ }^{2}$, M. De Pascale ${ }^{3}$, T. Masseron ${ }^{2}$, \\ N. Prantzos ${ }^{4}$, and Š. Mikolaitis ${ }^{5}$ \\ 1 Université Côte d' Azur, Laboratoire Lagrange, Observatoire de la Côte d'Azur, CNRS, Bd de l'Observatoire, CS 34229, 06304 Nice \\ Cedex 4, France \\ 2 Institute of Astronomy, University of Cambridge, Madingley Road, Cambridge CB3 OHA, UK \\ 3 INAF-Osservatorio Astronomico di Padova, Vicolo Osservatorio 5, 35122 Padova, Italy \\ ${ }^{4}$ Institut d'Astrophysique de Paris, UMR 7095 CNRS, Université P. \& M. Curie, 98bis Bd. Arago, 75104 Paris, France \\ 5 Institute of Theoretical Physics and Astronomy, Vilnius University, Saulètekio al. 3, 10222 Vilnius, Lithuania
}

Received 13 May 2016 / Accepted 16 July 2016

\begin{abstract}
Context. The chemical evolution of lithium in the Milky Way represents a major problem in modern astrophysics. Indeed, lithium is, on the one hand, easily destroyed in stellar interiors, and, on the other hand, produced at some specific stellar evolutionary stages that are still not well constrained.

Aims. The goal of this paper is to investigate the lithium stellar content of Milky Way stars in order to put constraints on the lithium chemical enrichment in our Galaxy, in particular in both the thin and thick discs.

Methods. Thanks to high-resolution spectra from the ESO archive and high quality atmospheric parameters, we were able to build a massive and homogeneous catalogue of lithium abundances for 7300 stars derived with an automatic method coupling, a synthetic spectra grid, and a Gauss-Newton algorithm. We validated these lithium abundances with literature values, including those of the Gaia benchmark stars.

Results. In terms of lithium galactic evolution, we show that the interstellar lithium abundance increases with metallicity by 1 dex from $[\mathrm{M} / \mathrm{H}]=-1$ dex to +0.0 dex. Moreover, we find that this lithium ISM abundance decreases by about 0.5 dex at super-solar metalllicity. Based on a chemical separation, we also observed that the stellar lithium content in the thick disc increases rather slightly with metallicity, while the thin disc shows a steeper increase. The lithium abundance distribution of $\alpha$-rich, metal-rich stars has a peak at $A_{\mathrm{Li}} \sim 3$ dex.

Conclusions. We conclude that the thick disc stars suffered of a low lithium chemical enrichment, showing lithium abundances rather close to the Spite plateau while the thin disc stars clearly show an increasing lithium chemical enrichment with the metallicity, probably thanks to the contribution of low-mass stars.
\end{abstract}

Key words. Galaxy: abundances - Galaxy: stellar content - Galaxy: formation - stars: abundances

\section{Introduction}

Nowadays, the lithium chemical element represents a major centre of interest because its chemical evolution history in stars and in the Milky Way is still uncertain. On the one hand, according to the Standard Big Bang Nucleosynthesis model (SBBN), the primordial lithium abundance is predicted to be $A_{\mathrm{Li}} \sim 2.6 \mathrm{dex}$ (Spergel et al. 2003); however, this primordial lithium abundance is in strong disagreement with the photospheric lithium abundance measured in old metal-poor dwarfs $\left(A_{\mathrm{Li}}=2.2 \mathrm{dex}\right)$ known as the Spite plateau (Spite \& Spite 1982). On the other hand, the lithium meteoritic abundance is known to be $A_{\mathrm{Li}}=$ 3.26 dex (Lodders et al. 2009) indicating a certain enrichment since the Big Bang, whereas the solar lithium photospheric abundance is sensitively lower, i.e. $A_{\mathrm{Li}}=1.05$ dex, (Grevesse et al 2007) highlighting internal destruction. Indeed, lithium is easily destroyed in stellar interiors $\left(T \sim 2 \times 10^{6} \mathrm{~K}\right)$ by $(\mathrm{p}, \alpha)$ reactions

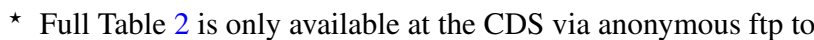
cdsarc.u-strasbg.fr (130.79.128.5) or via http://cdsarc.u-strasbg.fr/viz-bin/qcat?]/A+A/595/A18 and, because of mixing events, lithium can be strongly depleted at the stellar surface.

Lithium can also be produced in the interstellar medium (ISM) via spallation by Galactic cosmic rays (GCC; Reeves 1970) and at very specific phases of the stellar evolution: core-collapse supernovae (CCSN; Hartmann et al. 1999); novae (Arnould \& Norgaard 1975), as supported by recent observation of Tajitsu et al. (2015); and low-mass giants via cool bottom burning (Sackmann \& Boothroyd 1999), as observed first by Wallerstein \& Sneden (1982). Finally, asymptotic giant branch stars (AGB) can produce lithium via hot bottom burning (Sackmann \& Boothroyd 1992; Abia et al. 1999) as first observed by Mac Kellar (1940).

Nevertheless, even if several production sites are known, these stellar yields are still not well constrained. For example, adopting yields from Woosley et al. (1990), Travaglio et al. (2001) estimated that CCSN could produce $40 \%$ of the meteoritic abundance, while their contribution falls down to $10 \%$ when adopting hydrodynamical models (Heger et al. 2000). Building chemical evolution models of lithium in the Milky Way 
is thus not an easy task (Romano et al. 2001; Prantzos 2012). Moreover, when comparing observations to chemical evolution models, the lack of good age estimates does not allow us to study the lithium evolution with stellar age (Lambert \& Reddy 2004; Delgado Mena et al. 2015).

In order to investigate the lithium evolution in the Milky Way, one needs a statistically robust and homogeneous sample, such that a large metallicity domain can be covered. Up to now, very few studies presented small or inhomogeneous samples of few hundreds of stars (Lambert \& Reddy 2004; Ramírez et al. 2012; Delgado Mena et al. 2015). To provide more robust information on the lithium evolution in the Galaxy, we therefore performed a chemical study of the lithium behaviour from a homogeneous and very large catalogue of abundances created from the ESO archive without any external addition of smaller catalogues. This study is placed in the context of the AMBRE project (de Laverny et al. 2013b). The automatic determination of lithium has been performed for 7300 non-rotating and assumed non-binary stars, covering a wide range of metallicity.

The paper is organized as follows: in Sect. 2 we present the spectroscopic data used for our analysis while in Sect. 3 we detail our automatic method of lithium derivation. The AMBRE catalogue of lithium abundances is presented in Sect. 4. We validate our lithium measurements in Sect. 5. Finally, the lithium evolution in the Galaxy is discussed in Sect. 6 in the context of recent chemical evolution models of the Milky Way taking the thin to thick dichotomy into account. We conclude this work in Sect. 7.

\section{Observational data set from the AMBRE project}

This lithium study is based on spectroscopic data from the AMBRE project. The goal of this project is to parametrize the HARPS, FEROS, UVES, and GIRAFFE spectral archives (de Laverny et al. 2013b), providing robust automatic determinations of the radial velocity $\left(V_{\mathrm{rad}}\right)$, effective temperature $\left(T_{\text {eff }}\right)$, surface gravity $(\log (g))$, metallicity $([\mathrm{M} / \mathrm{H}])$, and global $\alpha$ enrichment with respect to iron $([\alpha / \mathrm{Fe}])$ together with their associated errors. In the present paper, we work with the first three samples that have already been parametrized: 3628 UVES spectra in the Red580 setup containing the Li feature at $6708 \AA$ (Worley et al. 2016), 89183 HARPS spectra (De Pascale et al. 2014), and 5981 FEROS spectra (Worley et al. 2012). These analysed samples were built from the spectra corresponding to stars with $+1 \leq \log (g) \leq+5 \mathrm{~cm} \mathrm{~s}^{-2}$ and a quality flag lower or equal to 1 (see e.g. Worley et al. 2012 for details on this label). The HARPS sample actually consists of repeated spectra for most of stars, thus the number of analysed stars is much lower. The typical errors on $T_{\text {eff }}, \log (g)$ and $[\mathrm{M} / \mathrm{H}]$ are $[108 \mathrm{~K}$, $0.16 \mathrm{~cm} \mathrm{~s}^{-2}, 0.10 \mathrm{dex}$ ] for UVES, [ $93 \mathrm{~K}, 0.26 \mathrm{~cm} \mathrm{~s}^{-2}, 0.08 \mathrm{dex}$ ] for HARPS, and $\left[120 \mathrm{~K}, 0.20 \mathrm{~cm} \mathrm{~s}^{-2}, 0.10 \mathrm{dex}\right]$ for FEROS. In the following, we also use the AMBRE estimates of the signalto-noise ratio $(\mathrm{S} / \mathrm{N})$ and the FWHM of the cross-correlation function used to derive $V_{\text {rad }}$ for a given star $\left(\mathrm{FWHM}_{\mathrm{CCF}}\right)$.

\section{Automatic lithium abundance analysis}

The lithium abundances of the AMBRE spectra were automatically derived via an optimization method, by coupling a precomputed synthetic spectra grid and the GAUGUIN GaussNewton algorithm (Bijaoui et al. 2012). We detail here the steps of our procedure.

\subsection{The high-resolution synthetic spectra grid around the Li doublet}

As the AMBRE stellar sample covers a wide range of atmospheric parameters, a careful examination of atomic and molecular contributions to the emerging spectrum was performed to build the line list for the synthetic spectra grid computation.

We first started with an atomic line list over $15 \AA$ from the Vienna Atomic Line Database (VALD3; Kupka et al. 1999, 2000). The line lists of nine molecular species were also taken into account: CN (Sneden et al. 2014), TiO (Plez, priv. comm.), C2 (Brooke et al. 2013; Ram et al. 2014), CH (Masseron et al. 2014), ZrO (Plez, priv. comm.), OH (Masseron, priv. comm), $\mathrm{CaH}$ (Plez, priv. comm.), $\mathrm{VO}$ (Plez, priv. comm.), and $\mathrm{SiH}$ (Kurucz 1992). We focused on a small wavelength range around the ${ }^{7} \mathrm{Li}$ doublet (located at $6707.8 \AA$ ), from 6707.0 to $6708.5 \AA$, at $R=40000$. This narrow domain is sufficiently wide to measure large lithium features with our automatic procedure of lithium derivation. For the ${ }^{7} \mathrm{Li}$ doublet at $6708 \AA$, we adopted the hyper-fine structure, consisting of six components. The wavelengths were taken from Sansonetti et al. (1995), while the oscillator strengths come from the calculations of Yan et al. (1998).

Our final line list was carefully calibrated with the Sun and Arcturus. We used the solar spectrum from Neckel (1999) and the Hinkle Arcturus Atlas (Hinkle et al. 2003). For Arcturus, we considered the atmospheric parameters from Ramírez \& Allende Prieto (2011). We only calibrated the main atomic lines contributing to the opacity (see Table 1). We increased the oscillator strength for the V I line at $\lambda=6707.518 \AA$ compared to previous studies (about $1.1 \mathrm{dex}$ with respect to Ghezzi et al. 2009) to reproduce the red part of the blend in Arcturus, as this VI line has a small contribution in the Sun. As another source of opacity is clearly missing in the Sun at $\lambda \approx 6708.0 \AA$, we included an additional line as already assumed in several previous works. Müller et al. (1975) first proposed adding a Si I line with $\chi_{\mathrm{e}}=6.00 \mathrm{eV}$ to match the solar spectrum while later Mandell et al. (2004) independently tested the two lines Ti I and Ti II. We rejected the Ti contribution that clearly overestimates the strength of the feature in Arcturus, whereas the Si I line provided a satisfying fit for both reference stars. The final fit between the observed solar and Arcturus spectra were very good with a typical flux discrepancy equal to 0.07 and $0.23 \%$ per pixel, respectively.

Based on this line list, a specific synthetic spectra grid was computed using the MARCS atmosphere models (Gustafsson et al. 2008) and the LTE TURBOSPECTRUM code (Plez 2012). Five dimensions were considered for this grid: $T_{\text {eff }}, \log (g),[\mathrm{M} / \mathrm{H}],[\alpha / \mathrm{Fe}]$, and $A_{\mathrm{Li}}{ }^{1}$. The ranges of the atmospheric parameters are those of the AMBRE grid, $3000 \leq$ $T_{\text {eff }} \leq 8000 \mathrm{~K}$ (in steps of $200 \mathrm{~K}$ below $4000 \mathrm{~K}$ and $250 \mathrm{~K}$ above), $+0 \leq \log (g) \leq+5.5 \mathrm{~cm} \mathrm{~s}^{-2}$ (in steps of $0.5 \mathrm{~cm} \mathrm{~s}^{-2}$ ), $-5 \leq[\mathrm{M} / \mathrm{H}] \leq+1$ dex (see Fig. 2 in de Laverny et al. 2012, for more details on the steps in $[\mathrm{M} / \mathrm{H}])$, whereas the variation in $A_{\mathrm{Li}}$ ranges from -1 to +4 dex with a step of +0.2 dex (26 different values of $A_{\mathrm{Li}}$ ).

The microturbulence velocity $(\xi)$ was included in the grid computation by adopting $\xi$ varying as a function of $T_{\text {eff }}, \log (g)$, and $[\mathrm{Fe} / \mathrm{H}]$ as adopted in the Gaia-ESO Survey (Bergemann et al., in prep., based on $\xi$ determinations from literature samples). The total number of synthetic spectra is 358335 , computed on a wavelength range of $15 \AA$, centred on the lithium doublet

\footnotetext{
$1 \quad A_{\mathrm{Li}}=\log \epsilon(\mathrm{Li})$; both notations are in logarithmic scale of number of
} atoms where $\log \epsilon(\mathrm{H})=A_{\mathrm{H}}=12$. 
Table 1. Main atomic lines around the ${ }^{7} \mathrm{Li}$ doublet.

\begin{tabular}{cccc}
\hline \hline Element & $\begin{array}{c}\text { Wavelength } \\
(\AA)\end{array}$ & $\begin{array}{c}\chi_{\mathrm{e}} \\
(\mathrm{eV})\end{array}$ & $\log g f$ \\
\hline Fe I $_{\text {I }}$ & 6707.070 & 5.273 & $\mathbf{- 2 . 4 8 0}$ \\
Fe I & 6707.172 & 5.538 & $\mathbf{- 2 . 6 0 0}$ \\
Fe I & 6707.431 & 4.608 & $\mathbf{- 2 . 1 7 5}$ \\
Sm II & 6707.473 & 0.933 & -1.910 \\
V I & 6707.518 & 2.743 & $\mathbf{- 0 . 8 0 0}$ \\
Cr I & 6707.596 & 4.208 & -2.625 \\
Li I & 6707.756 & 0.000 & -0.428 \\
Li I & 6707.768 & 0.000 & -0.206 \\
Li I & 6707.907 & 0.000 & -1.509 \\
Li I & 6707.908 & 0.000 & -0.807 \\
Li I & 6707.919 & 0.000 & -0.807 \\
Li I & 6707.920 & 0.000 & -0.807 \\
Si I & 6708.023 & 6.000 & $\mathbf{- 2 . 8 2 0}$ \\
V I & 6708.094 & 1.218 & $\mathbf{- 2 . 8 1 0}$ \\
Ce II & 6708.099 & 0.701 & -2.120 \\
Fe I & 6708.282 & 4.988 & $\mathbf{- 2 . 6 3 0}$ \\
Fe I & 6708.347 & 5.486 & -2.506 \\
\hline
\end{tabular}

Notes. Astrophysically calibrated $\log g f$ values are highlighted in boldface.

at $6708 \AA$ with a sampling of $0.004 \AA$ and a spectral resolution thatis higher than 150000 .

\subsection{Preparing the set of synthetic and observed spectra}

One of the main characteristics of our method is that we do not synthesize on the fly model spectra to fit the observed spectrum. We interpolate the pre-computed 5D synthetic spectra grid of Sect. 3.1 at the atmospheric parameters of the target derived within the AMBRE project to prepare a small set of interpolated synthetic spectra for a direct comparison with the observation. These spectra are interpolated in a first step. This interpolating spectra procedure allows us to derive abundances very quickly for large sets of observations.

More precisely, we build a set of interpolated synthetic spectra, composing a $1 \mathrm{D}$ grid $S\left(A_{\mathrm{Li}}, \lambda\right)$ on the lithium abundance. Practically speaking, a cubic interpolation of the 5D synthetic spectra grid was performed at the four atmospheric parameters of the observed star $T_{\text {eff }}^{\star}, \log (g)^{\star},[\mathrm{Fe} / \mathrm{H}]^{\star}$, and $[\alpha / \mathrm{Fe}]^{\star}$, resulting in the $1 \mathrm{D}$ grid $S\left(A_{\mathrm{Li}}, \lambda\right)$. As the variation in flux can be non-linear, we performed a Catmull-Rom interpolation based on Bézier curves. For a given lithium abundance of the 1D grid, the corresponding synthetic interpolated spectrum is a combination of $4^{4}=256$ synthetic spectra because the four closest spectra in each parameter dimension $\left(T_{\mathrm{eff}}, \log (g),[\mathrm{M} / \mathrm{H}]\right.$, and $\left.[\alpha / \mathrm{Fe}]\right)$ are considered and weighted.

For stars with parameters close to the edges of the synthetic spectra grid, the 256 spectra are not systematically found and a simple linear interpolation is carried out. Roughly half of the AMBRE stars are interpolated linearly. The resulting 1D grid $S\left(A_{\mathrm{Li}}, \lambda\right)$ in lithium abundance at $T_{\mathrm{eff}}^{\star}, \log (g)^{\star},[\mathrm{Fe} / \mathrm{H}]^{\star}$, and $[\alpha / \mathrm{Fe}]^{\star}$ varies from -1 to +4 dex and is composed of 26 model spectra.

Concerning the observed spectra $O(\lambda)$, their resolution was degraded to 40000 considering an instrumental Gaussian profile. This value was chosen as being the lowest spectral resolution of the three spectrographs FEROS, UVES, and HARPS. This allows us to analyse all the AMBRE spectra in a very homogeneous way. Respecting the Shannon theorem, we re-sampled these spectra to a pixel size of $0.05 \AA$. We applied the same
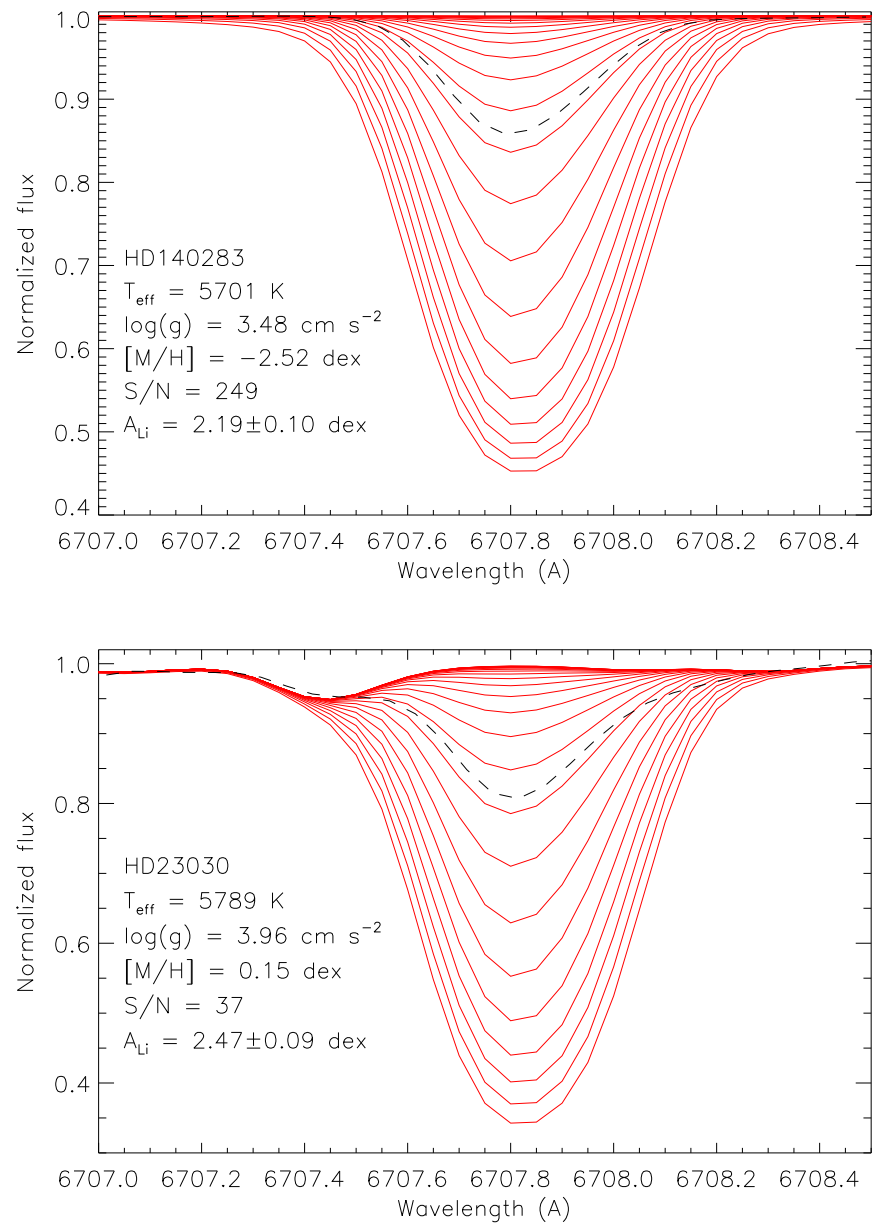

Fig. 1. Observed spectrum (dashed line) of HD 140283 (top) and HD 23030 (bottom) at $R=40000$. The corresponding 1D synthetic spectra grid with lithium variations from -1 to 4 dex and a step of 0.2 dex, with the grid interpolated at the atmospheric parameters of both stars, is shown in red.

convolution and re-sampling to the synthetic spectra grid. Then, to put the spectra in the same rest frame, we performed the radial velocity correction with the value provided by the AMBRE project.

Finally, an automatic adjustment of the continuum was performed on the observed spectrum. For that purpose, we adopted an interpolated spectrum at the atmospheric parameters of the star. We removed the line features by $\sigma$-clipping, and a ratio between the synthetic flux and the observed flux is computed over a spectral range of $15 \AA$, centred on the lithium doublet. This ratio is consecutively fitted by a third order polynomial function, $\sigma$-clipped, and then fitted a second time. Finally, the observed spectra are divided by the polynomial fit to adjust their continuum. This step was validated automatically by checking that the normalization is consistent over a much broader spectral range of $100 \AA$. Systematic errors on $A_{\mathrm{Li}}$ determination due to automatic continuum placement were not estimated, but should be negligible, for example compared to the errors of the effective temperature and taking into account that our sample is mainly composed of high $\mathrm{S} / \mathrm{N}$ spectra.

We show two typical examples of 1D grids and observed spectra in Fig. 1, for HD 140283 and HD 23030, observed with a S/N of 249 and 37 , respectively. 


\subsection{Derivation of the lithium abundances with GAUGUIN}

From the 1D lithium grid $S\left(A_{\mathrm{Li}}, \lambda\right)$ described above, we compute a quadratic distance $D\left(A_{\mathrm{Li}}, \lambda\right)$ between the observed spectrum $O(\lambda)$ and each point of the grid $S\left(A_{\mathrm{Li}}, \lambda\right)$ over a wavelength range of $1.5 \AA$ centred on the lithium doublet. The minimum of $D\left(A_{\mathrm{Li}}, \lambda\right)$ provides a first guess $A_{\mathrm{Li}}^{0}$ of the solution, i.e. the closest point of the grid. Then, this first guess is optimized via the Gauss-Newton algorithm GAUGUIN (Bijaoui et al. 2012). For that purpose, from $S\left(A_{\mathrm{Li}}, \lambda\right)$, a synthetic spectrum is interpolated at $A_{\mathrm{Li}}^{0}$ and the correction computed by GAUGUIN is then

$\delta_{A_{\mathrm{Li}}}=\left(\mathbf{J}^{T} \mathbf{J}\right)^{-1} \mathbf{J}^{T}\left[O(\lambda)-S\left(A_{\mathrm{Li}}^{0}, \lambda\right)\right]$,

where $\mathbf{J}$ is the Jacobian matrix $\left.\left[\partial S\left(A_{\mathrm{Li}}^{0}, \lambda\right)\right) / \partial A_{\mathrm{Li}}\right]$. As the curve of growth is highly non-linear with the abundance, the flux in the lithium line varies non-linearly as well and the Catmull-Rom interpolation is again used to build the interpolated flux and derivatives. The algorithm stops when the distance is minimal between the interpolated spectrum $S\left(A_{\mathrm{Li}}, \lambda\right)$ and $O(\lambda)$. A wavelength range (large enough to safely measure the highest abundances) of $1.5 \AA$ centred on the lithium doublet is considered for this operation. Upper limits are provided when the lithium feature is too weak with respect to the $\mathrm{S} / \mathrm{N}$ of the spectrum. On the other hand, we can detect lower limits for $A_{\mathrm{Li}} \geq 4$ dex.

The simple application of GAUGUIN, which is basically lower than $10 \mathrm{~ms}$, is very fast for a single spectral line. This completely automatic procedure (including all the synthetic spectra preparation described in Sect. 3.2) is fast and adapted for massive spectroscopic surveys. Basically, a single Li line abundance can be derived in $\approx 1.5 \mathrm{~s}$. The pipeline was implemented combining IDL and $\mathrm{C}++$ languages.

The errors on $A_{\mathrm{Li}}$ were estimated by propagating the errors on the three atmospheric parameters $\left\{T_{\mathrm{eff}}^{\star}, \log (g)^{\star},[\mathrm{M} / \mathrm{H}]^{\star}\right\}$ provided by AMBRE and summing them quadratically (see Sect. 4 and Fig. 2). The error on the lithium measurement is dominated by the error on the effective temperature. Furthermore, we checked that a typical error of $1 \mathrm{~km} \mathrm{~s}^{-1}$ on the radial velocity leads to a negligible error on $A_{\mathrm{Li}}$. Also, by generating Sun and Arcturus synthetic spectra for different values of $\xi$ and $A_{\mathrm{Li}}$ from 1 to $3 \operatorname{dex}(R=40000)$, we checked the impact of a pessimistic error on $\xi$ of $1 \mathrm{~km} \mathrm{~s}^{-1}$ for the dwarfs and $2 \mathrm{~km} \mathrm{~s}^{-1}$ for the giants. For the Sun, the error defined by $e_{A_{\mathrm{Li}}} / A_{\mathrm{Li}}$ is largely below $1 \%$ whatever the value of $A_{\mathrm{Li}}$ and can thus be neglected compared to the error contribution of the atmospheric parameters. For Arcturus, the error is also low (4\% at 2 dex and 5\% at 3 dex), which is well below the typical errors caused by the other atmospheric parameters for the AMBRE giants. This $\xi$ error contribution has thus been neglected in the derivation of the total error.

Finally, we assume, for this lithium analysis, that all the targets are single stars since binary detection is not a part of the AMBRE parametrization pipeline.

\subsection{Non-LTE corrections of the AMBRE/Li abundances}

As the AMBRE stellar sample and synthetic spectra grid cover a wide range of atmospheric parameters, it is important to take possible non-local thermodynamic equilibrium (NLTE) effects on the AMBRE/Li abundances into account. For that purpose, we adopted the NLTE corrections presented by Lind et al. (2009a) to estimate the corrections that we have to apply to our Li measurements. For these NLTE corrections, we assumed $[\mathrm{M} / \mathrm{H}]=+0$ dex for stars with $[\mathrm{M} / \mathrm{H}]>0$ dex because the dependence with $[\mathrm{M} / \mathrm{H}]$ is low (K. Lind, priv. comm.). Moreover, we adopted the NLTE corrections at $[\mathrm{M} / \mathrm{H}]=-3$ dex for the few metal-poor stars with $[\mathrm{M} / \mathrm{H}]<-3 \mathrm{dex}$, as adopted for example by Sbordone et al. (2010). We also did not publish the NLTE corrections for stars outside of the Lind et al. ranges $(2 \%$ of the AMBRE catalogue), mostly when $T_{\text {eff }}<4000 \mathrm{~K}$. Finally, for the most lithium-poor stars with $A_{\mathrm{Li}}$ lower than the lowest value of the Lind et al. LTE curve-of-growth ( -0.3 dex, variable with $T_{\text {eff }}$ ), no corrections were computed; this subsample represents $9 \%$ of the AMBRE catalogue. The resulting corrections are presented in Sect. 4 (see Table 2, Col. 7) for $89 \%$ of the AMBRE catalogue.

\section{The AMBRE catalogue of lithium abundances}

The AMBRE/Li catalogue is presented in Table 2. We first looked for repeated observations separately in each of the UVES, HARPS, and FEROS samples. For UVES and FEROS, we performed a cross-match on the spectra coordinates with a radius of 2 and 10 arcsec on the sky, leading to remaining samples composed of 1031, and 3526 stars, respectively. For a given star with several spectra collected with the same spectrograph, we chose the spectrum with the best atmospheric parameters in terms of the AMBRE $\chi^{2}$ quality flag (see Worley et al. 2012), leading to the best lithium measurement. For HARPS, as the number of repeated spectra for a given star can be huge with different target stars very close to each other, we adopted the sample of 4355 stars from Mikolaitis et al. (in prep.) based on a search of both coordinates and atmospheric parameters differences. The last step to merge these UVES, HARPS, and FEROS samples together was to identify stars possibly observed with two or three spectrographs with a new coordinate cross-match on a radius of 10 arcsec. In this case, for a given star, we selected its spectrum with the best parameters in terms of $\chi^{2}$ as explained above. The final working AMBRE catalogue is then composed of 7821 stars, subdivided into 3301 FEROS, 878 UVES, and 3642 HARPS stars.

We are aware that the synthetic spectra grid was computed with no rotation, assuming that the stars are slow rotators. In order to estimate the impact of the rotation on the derived AM$\mathrm{BRE} / \mathrm{Li}$ abundances, we artificially broadened solar and Arcturus synthetic spectra for different $A_{\mathrm{Li}}$ values. Using typical $v \sin (i)$ values lower than $10 \mathrm{~km} \mathrm{~s}^{-1}$ for Arcturus and $15 \mathrm{~km} \mathrm{~s}^{-1}$ for the Sun, the estimated errors on $A_{\mathrm{Li}}$ are found to be lower than errors due to the atmospheric parameters. We therefore adopted these $v \sin (i)$ values as the largest acceptable values to derive $A_{\mathrm{Li}}$. Thus, since AMBRE does not provide the rotational velocity and only provides the FWHM of the crosscorrelation function $\left(\mathrm{FWHM}_{\mathrm{CCF}}\right)$, we looked for a relation between $v \sin (i)$ and $\mathrm{FWHM}_{\mathrm{CCF}}$ for the AMBRE spectra. First, based on rotational velocities for stars from the FEROS sample (de Laverny et al. 2013a), we searched for a limit in terms of $\mathrm{FWHM}_{\mathrm{CCF}}$ (that is sensitively correlated to $v \sin (i)$ ) that was valid over the whole atmospheric parameter range. We established a limit at $F W H M_{\mathrm{CCF}}=20 \mathrm{~km} \mathrm{~s}^{-1}$ for FEROS targets. We thus removed stars with higher FWHM $_{\mathrm{CCF}}(320$ stars, $10 \%$ of the FEROS sample). Concerning the HARPS targets, showing lower FWHM $\mathrm{CCF}_{\mathrm{CF}}$ because of the larger resolution, we applied the same strategy as for FEROS, but based on $v \sin (i)$ estimations from Mikolaitis et al. (in prep.). The established limit is $F W H M_{\mathrm{CCF}}=15 \mathrm{~km} \mathrm{~s}^{-1}$ (removing then 150 stars, $4 \%$ of the HARPS sample). For UVES stars, the FWHM FCF $_{\text {distribution }}$ shows intermediate values between HARPS and FEROS. As we do not have any $v \sin (i)$ determinations for these stars, we applied 
Table 2. Identifier, spectrograph $(\mathrm{U}=\mathrm{UVES}, \mathrm{H}=\mathrm{HARPS}, \mathrm{F}=$ FEROS), lithium abundances, and NLTE-corrections (defined by $\left.\Delta_{\mathrm{NETL}}=A_{\mathrm{Li}}^{\mathrm{NLTE}}-A_{\mathrm{Li}}^{\mathrm{LTE}}\right)$ of the AMBRE catalogue of lithium measurements.

\begin{tabular}{lccc}
\hline \hline TARGNAME & Spectro & $\begin{array}{c}A_{\mathrm{Li}}^{\mathrm{LTE}} \\
(\mathrm{dex})\end{array}$ & $\begin{array}{c}\Delta_{\text {NETL }} \\
(\mathrm{dex})\end{array}$ \\
\hline HD 162396 & $\mathrm{H}$ & $+2.32 \pm 0.08$ & -0.01 \\
HD 199288 & $\mathrm{H}$ & $+0.97 \pm 0.09$ & +0.01 \\
HD 90422 & $\mathrm{H}$ & $+1.61 \pm 0.08$ & -0.01 \\
HD 128167 & $\mathrm{H}$ & $<+1.40$ & - \\
HD 091324 & $\mathrm{H}$ & $+1.95 \pm 0.07$ & -0.01 \\
HD 215257 & $\mathrm{H}$ & $+2.27 \pm 0.08$ & -0.03 \\
HD 211998 & $\mathrm{H}$ & $+1.20 \pm 0.10$ & +0.03 \\
$\ldots$ & $\ldots$ & $\ldots$ & $\ldots$ \\
\hline
\end{tabular}

a very pessimistic cut at $F W H M_{\mathrm{CCF}}=15 \mathrm{~km} \mathrm{~s}^{-1}$ (removing then 79 stars, $9 \%$ of the UVES sample), as that adopted for the higher HARPS spectra resolution. For all these rotating stars, we decided not to publish their $A_{\mathrm{Li}}$ abundance.

As a consequence, the AMBRE/Li catalogue finally contains 7272 stars $^{2}$, i.e. $\sim 93 \%$ of the stars for which we derived $A_{\mathrm{Li}}$, subdivided into 2981 FEROS, 799 UVES, and 3492 HARPS stars. Among these, 6479 stars have NLTE corrections. Up to now, this catalogue is the largest ever created, and is more than one order of magnitude larger than previous studies (Delgado Mena et al. 2014, 2015, 353 and 326 dwarf stars, respectively; Ramírez et al. 2012, 671 dwarf stars; Liu et al. 2014378 giant stars). Moreover, this AMBRE/Li catalogue is perfectly homogeneous in terms of atmospheric parameters and $A_{\mathrm{Li}}$ determinations. The lithium measurements and NLTE corrections of these 7272 stars are presented in Table 2. Atmospheric parameters of the AMBRE/Li stars are available in Worley et al. $(2012,2016)$ and in De Pascale et al. (2014).

Together with $A_{\mathrm{Li}}$, we also provide the errors on $A_{\mathrm{Li}}$ in Table 2. We show these errors on $A_{\mathrm{Li}}$ in Fig. 2. We see that the error strongly increases for lower effective temperatures and tend to be lower for metal-poor stars for which the determinations are more robust (less blend). Almost $98 \%$ of the stars have an error that is lower than 0.3 dex, while $40 \%$ of the sample (the hottest one with $T_{\text {eff }}>5500 \mathrm{~K}$ ) have an error lower than $0.1 \mathrm{dex}$, which is low enough for conducting a detailed scientific application of our lithium measurements.

We also show in Fig. 3 the Hertzsprung-Russel diagram of this AMBRE catalogue of lithium abundances for slow rotators. Thanks to the binning in $[\mathrm{M} / \mathrm{H}]$, we clearly see that the statistics in each stellar population are high, from metal-poor to metal-rich stars.

We present the behaviour of $A_{\mathrm{Li}}^{\mathrm{NLTE}}$ with $T_{\text {eff }}$ in Fig. 4 . We see that cooler stars exhibit lower lithium abundances probably because of their deeper convective zone leading to higher destruction of lithium $(\sim 80 \%$ of the AMBRE sample is composed by dwarf stars). We also observe a strong correlation between the lower limits of lithium with the effective temperature. We can understand this trend by considering that for a given $\mathrm{S} / \mathrm{N}$ value, the detectable lithium feature is stronger at lower temperatures.

Finally, we detected the presence of lithium-rich giants in the AMBRE/Li catalogue. These stars will be studied in a work in preparation.

\footnotetext{
2 Stars with close coordinates but different atmospheric parameters were clearly tagged by adopting a different name in the AM$\mathrm{BRE} / \mathrm{Li}$ catalogue.
}

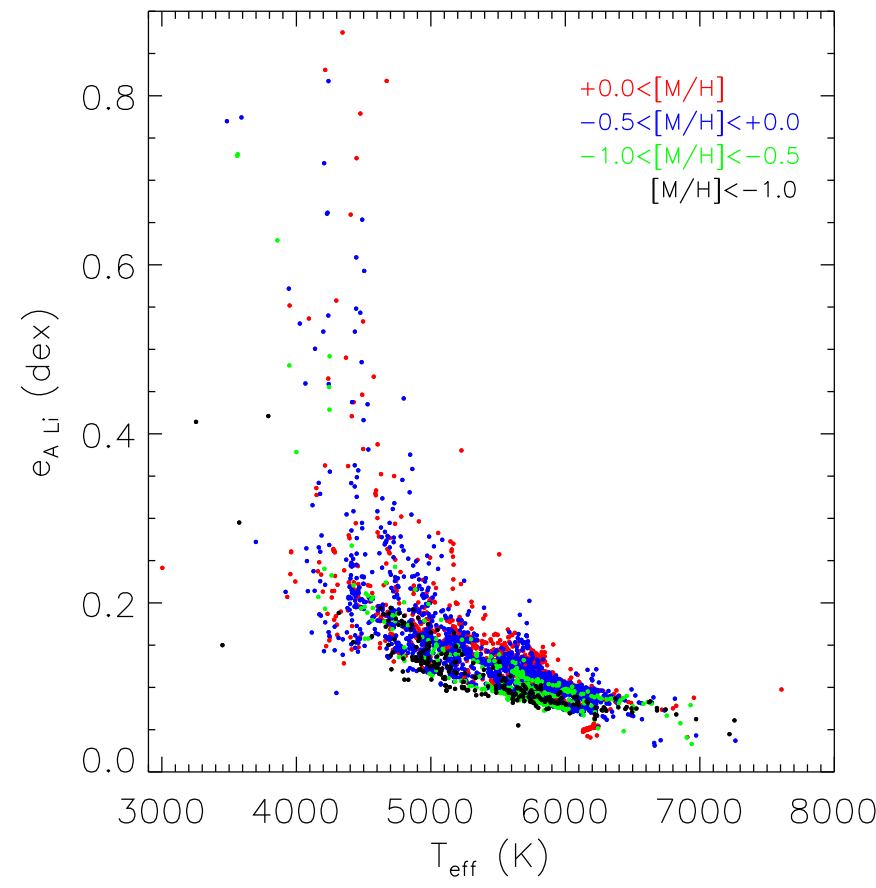

Fig. 2. Errors on $A_{\mathrm{Li}}^{\mathrm{ETL}}$ of the AMBRE catalogue of lithium measurements (excluding upper limits), colour-coded in bins of $[\mathrm{M} / \mathrm{H}]$.

\section{Internal and external validation}

\subsection{Internal comparison between HARPS and UVES targets}

Several stars from our catalogue have several abundances because these targets were originally observed by two spectrographs (we selected the best spectrum between both as explained before). This allows us to test the internal accuracy of our automatic method. For that purpose, we selected stars observed both with UVES and HARPS, resulting in a subsample of 117 dwarfs and giants (only very few stars were observed with HARPS and FEROS or UVES and FEROS, and we rejected these repeats because of insufficient statistics). This subsample covers a wide range of parameters, i.e. $4500<T_{\text {eff }}<6700 \mathrm{~K}, 2.5<\log (g)<$ $4.9 \mathrm{~cm} \mathrm{~s}^{-2}$, and $-0.9<[\mathrm{M} / \mathrm{H}]<+0.4$ dex. We compared both lithium abundances and upper limits (see Fig. 5).

On the one hand, we see that the 72 lithium measurements are in a very good agreement between both spectrographs with a mean difference of 0.05 dex and a standard deviation equal to 0.18 dex. This dispersion can be due to the standard deviation of the difference of the AMBRE effective temperatures between both spectrographs $(107 \mathrm{~K})$. The small bias can be related to the biases in $T_{\text {eff }}$ and $\log (g), 17 \mathrm{~K}$ and $-0.11 \mathrm{~cm} \mathrm{~s}^{-2}$, respectively between both spectrographs. The bias in $[\mathrm{M} / \mathrm{H}]$ is null. On the other hand, we see that the 43 upper limits of HARPS are systematically higher than the UVES upper limits. This can be well explained by the fact that, for this subsample, the average $\mathrm{S} / \mathrm{N}$ of HARPS targets is three times lower than for UVES; the detection limits are partly governed by the $\mathrm{S} / \mathrm{N}$ values. We also have two stars with lithium detection in UVES and upper limit with HARPS and a good consistency is found between them. We then conclude that our automatic method is robust since it is able to recover with good precision the lithium abundances when a star is observed by two different spectrographs. 

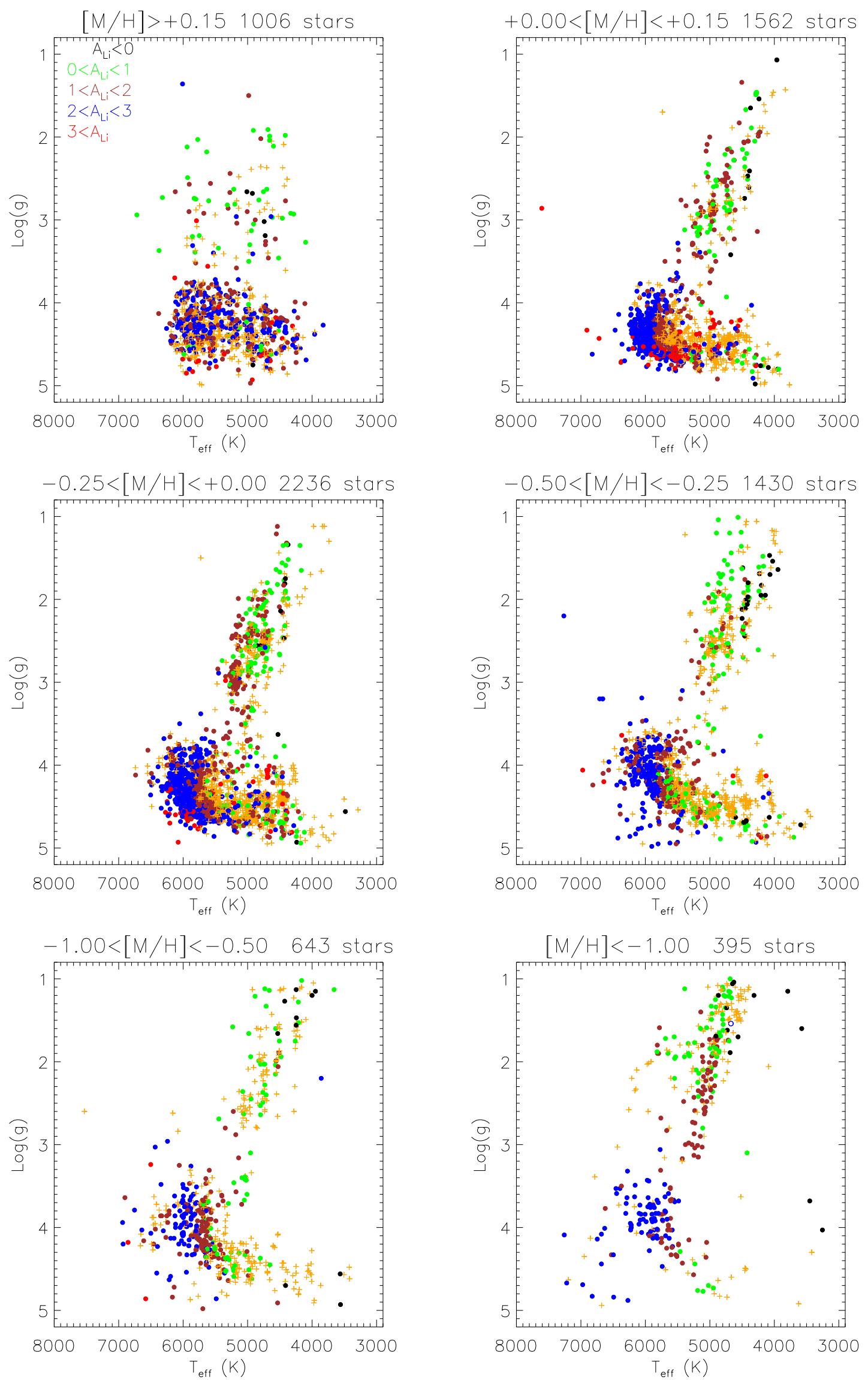

Fig. 3. $T_{\text {eff }}$ vs. $\log (g)$ for the AMBRE catalogue of lithium measurements colour-coded in bins of $A_{\mathrm{Li}}$ (LTE). Filled and open circles correspond to lithium detections and $A_{\mathrm{Li}}>4.2$ dex, respectively. Upper limits are represented by orange "+" symbol. 


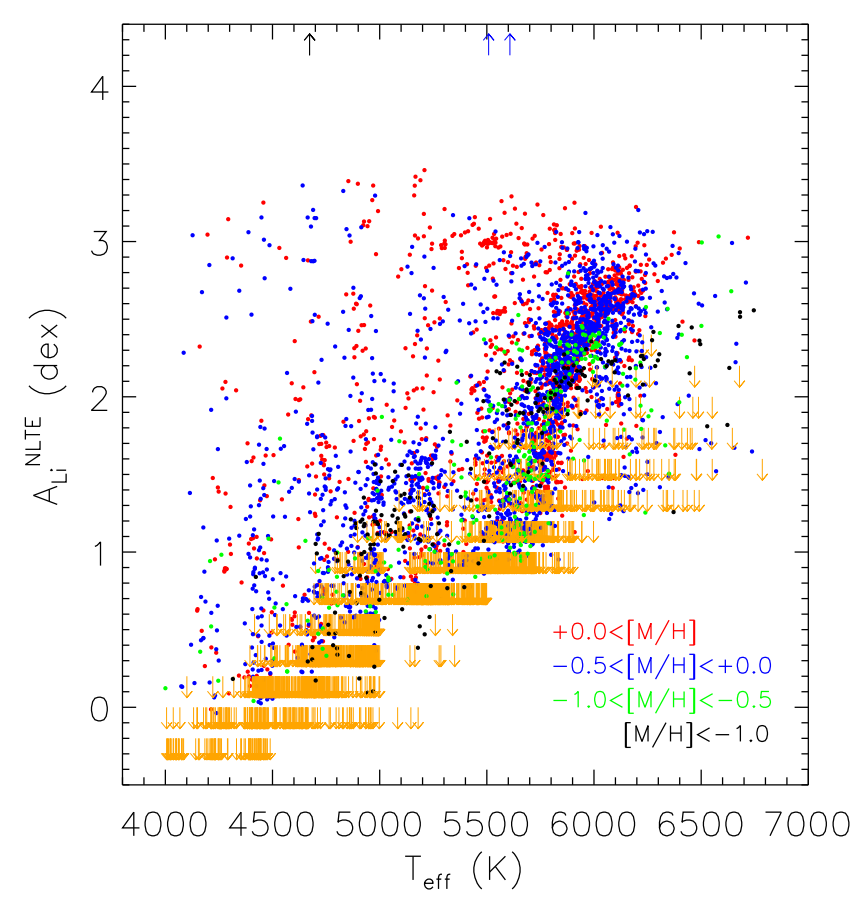

Fig. 4. $A_{\mathrm{Li}}^{\mathrm{NLTE}}$ vs. $T_{\text {eff }}$ with a colour-code in $[\mathrm{M} / \mathrm{H}]$. Upper and lower limits are symbolized by downwards and upwards arrows, respectively.

\subsection{Comparison with independent Li abundances}

\subsubsection{Ramírez et al. (2012)}

We first compare the AMBRE LTE lithium values with those of Ramírez et al. (2012) derived from UVES and HARPS spectra. The AMBRE/Li catalogue contains 74 dwarf stars with spectra already analysed by the authors, i.e. $11 \%$ of their sample. The atmospheric parameters of these stars in common are within $5300<T_{\text {eff }}<6300 \mathrm{~K}, 3.6<\log (g)<4.7 \mathrm{~cm} \mathrm{~s}^{-2}$ and $-1.0<[\mathrm{M} / \mathrm{H}]<+0.2$ dex. Ramírez et al. (2012) also adopted a different spectral synthesis code (MOOG2010; Sneden 1973) together with a different line list to determine their lithium abundances. The comparison of the derived lithium abundances is shown in Fig. 6 (left panel) for 59 stars, excluding upper limits. We see a good agreement between both studies with small bias and dispersion of -0.04 and 0.16 dex, respectively. This dispersion can be well explained by the dispersions of the difference in the adopted $T_{\text {eff }}, \log (g)$ and $[\mathrm{M} / \mathrm{H}]$ between both groups that we estimated to be $95 \mathrm{~K}, 0.18 \mathrm{~cm} \mathrm{~s}^{-2}$ and $0.08 \mathrm{dex}$, respectively. We also checked the measured upper limits of 25 stars and a very good consistency is also found.

\subsubsection{Delgado Mena et al. (2014)}

We compare the AMBRE LTE lithium values with those derived from HARPS spectra published by Delgado Mena et al. (2014). The AMBRE/Li catalogue contains 194 stars with HARPS spectra already analysed by Delgado Mena et al. (2014), i.e. 55\% of their sample. Apart from their line list, their adopted procedure to derive the lithium abundances is very close to that of Ramírez et al. (2012). The atmospheric parameters of these stars in common are within $5600<T_{\text {eff }}<5900 \mathrm{~K}, 3.6<\log (g)<$ $4.7 \mathrm{~cm} \mathrm{~s}^{-2}$ and $-1.0<[\mathrm{M} / \mathrm{H}]<+0.4 \mathrm{dex}$. The comparison of the derived lithium abundances is shown in Fig. 6 (middle panel) for 124 stars, excluding upper limits. We see a very good agreement between both studies with no bias and a small dispersion

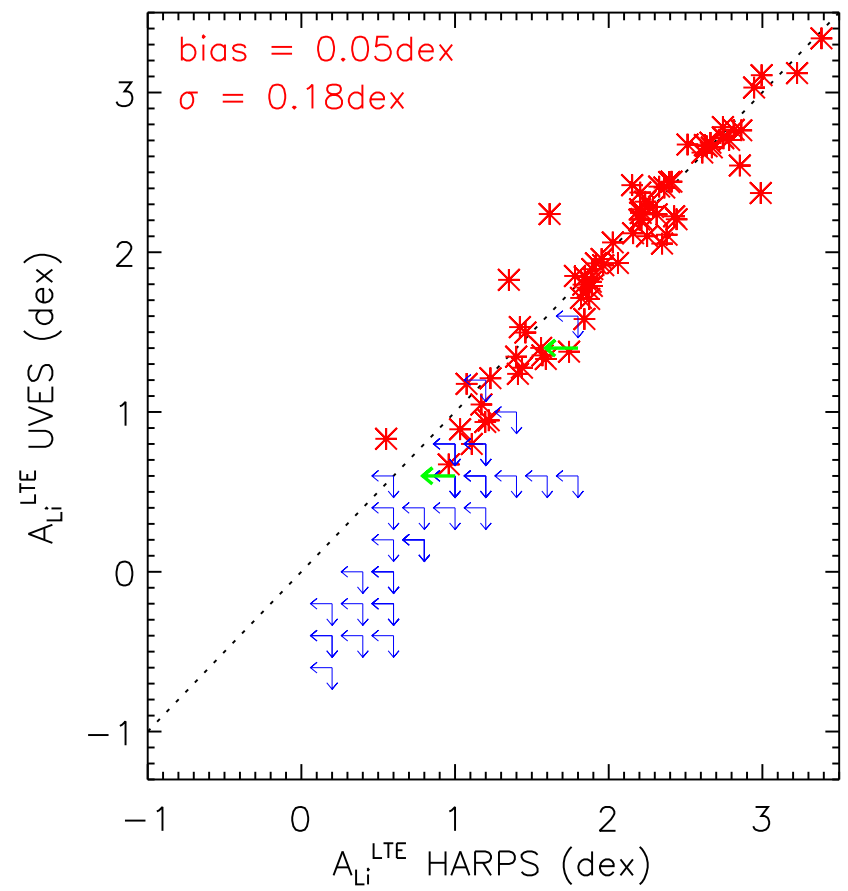

Fig. 5. Lithium abundances for a set of 117 dwarf and giant AMBRE stars observed both with UVES and HARPS. Lithium detections are shown in red, while upper limits are represented by blue and green arrows. Mean difference and standard deviation for the lithium detections are reported.

of 0.10 dex. This dispersion can be well explained by the dispersions of the difference in the adopted $T_{\text {eff }}, \log (g)$ and $[\mathrm{M} / \mathrm{H}]$ between both groups that we estimated to be $62 \mathrm{~K}, 0.17 \mathrm{~cm} \mathrm{~s}^{-2}$ and 0.06 dex, respectively. We clearly see, however, that this dispersion increases for lower $A_{\mathrm{Li}}$ values. At lower abundance and fixed other parameters, the blends contribution to the line profile starts to be stronger (particularly for cool stars) and a different blend treatment between both methods could explain such higher dispersion. We also checked the measured upper limits of 70 stars and a very good consistency is found again.

\subsubsection{Delgado Mena et al. (2015)}

We finally compare the AMBRE LTE lithium values with those of Delgado Mena et al. (2015) derived from HARPS spectra. There are 200 hot stars, i.e. $61 \%$ of their sample. The atmospheric parameters of these stars in common are within $5900<$ $T_{\text {eff }}<6700 \mathrm{~K}, 3.5<\log (g)<4.5 \mathrm{~cm} \mathrm{~s}^{-2}$, and $-1.0<[\mathrm{M} / \mathrm{H}]<$ +0.4 dex. The comparison of the derived lithium abundances is shown in Fig. 6 (right panel) for 179 stars, excluding upper limits. We see a good agreement between both studies with a bias of $-0.11 \mathrm{dex}$ and a small dispersion of 0.08 dex. This dispersion can be well explained by the dispersions of the difference in the adopted $T_{\text {eff }}, \log (g)$ and $[\mathrm{M} / \mathrm{H}]$ between both groups that we estimated to be, $78 \mathrm{~K}, 0.19 \mathrm{~cm} \mathrm{~s}^{-2}$ and $0.07 \mathrm{dex}$, respectively. The bias in $T_{\text {eff }}$ is not constant and increases with $T_{\text {eff. }}$. We also checked the measured upper limits of 21 stars and a very good consistency is found.

\subsection{Lithium abundance of the Gaia-benchmark stars}

As an additional check of our automatic procedure, we identified several Gaia-benchmark stars (Jofré et al. 2014a) in the 

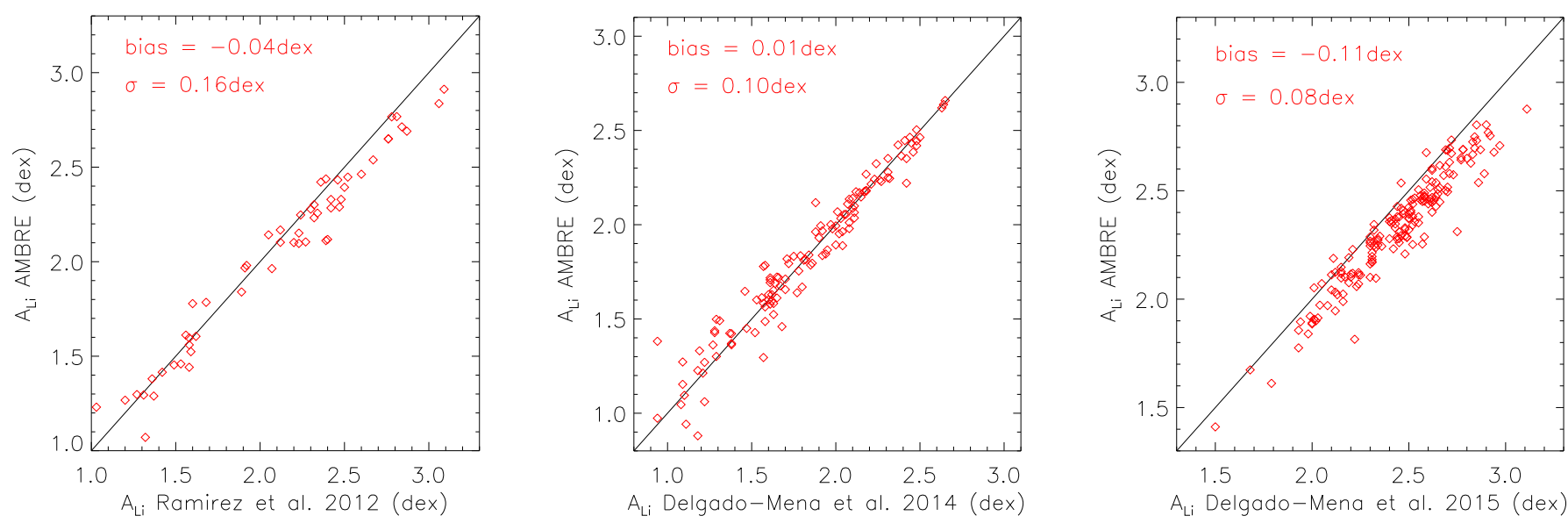

Fig. 6. Comparison between the LTE lithium abundances of the AMBRE/Li catalogue and the studies of Ramírez et al. (2012), Delgado Mena et al. (2014), and Delgado Mena et al. (2015).

AMBRE/Li catalogue. This identification was performed with the coordinates and TARGNAME identifier, resulting in 20 stars. We also measured $A_{\mathrm{Li}}$ for these same identified stars from the AMBRE spectra, but adopted the atmospheric parameters presented by recent studies reporting lithium abundances. We emphasize the fact that the literature studies are characterized by different spectral resolutions, $\mathrm{S} / \mathrm{N}$, atomic and molecular treatment, and also different techniques (e.g. spectral fitting and equivalent width analysis) for the abundance derivation. All these facts can be responsible for most of the (small) differences observed with respect to the literature. As presented in Fig. 7, we clearly see in the left panel that the comparison with published LTE lithium abundance is good. We measure a small bias (defined as the mean difference) of 0.05 dex and a dispersion $\sigma=0.23$ dex that can easily be explained by the different atmospheric parameters (and particularly $T_{\text {eff }}$ ) adopted in AMBRE and these literature studies. Indeed, when comparing the AM$\mathrm{BRE} / \mathrm{Li}$ abundances with the lithium abundances derived using the atmospheric parameters from the literature (middle panel), the agreement becomes very satisfactory with no bias and a smaller dispersion $\sigma=0.14$ dex. Finally, when adopting the effective temperature and surface gravity of Heiter et al. (2015) and metallicity of Jofré et al. (2014b), we see in Fig. 7 (right panel) that the derived lithium values are once again consistent with the AMBRE/Li abundances. The atmospheric parameters that are derived by Heiter et al. (2015) and Jofré et al. (2014b) are very similar to the independent determinations by the AMBRE project. These comparisons again confirm that the lithium abundances of the AMBRE/Li catalogue are reliable for a scientific exploitation.

\section{Tracing the lithium evolution in the Milky Way}

In this section, we propose to study the evolution of lithium enrichment in the Milky Way using the AMBRE catalogue of lithium abundances. We first investigate the stellar lithium content as a function of the metallicity used as a time tracer to constrain the abundance of the ISM in which they were formed. Then, we study the lithium enrichment in both the galactic thin and thick discs. To do this, we built a working sample from the catalogue presented in Sect. 4, rejecting stars with upper and lower limits in their lithium abundances. As we also want to avoid any possible lithium abundance variations caused by stellar evolution during the late stages, we only consider dwarf stars (defined hereafter as $\log (g) \geq 3.7 \mathrm{~cm} \mathrm{~s}^{-2}$ ). Finally, as the catalogue covers a wide range in effective temperature and metallicity, we selected targets with available NLTE corrections to compare the right lithium abundance correctly in a rather different type of stars. As a result, our working sample is composed of 3077 stars with minimal and median $\mathrm{S} / \mathrm{N}$ value of 15 and 82 , respectively.

\subsection{Lithium evolution with the metallicity}

The classical method to study the evolution of a chemical element in the Milky Way is to study its behaviour with the metallicity, used as a proxy of age (at least for $[\mathrm{M} / \mathrm{H}]<+0.0 \mathrm{dex}$ ). The main problem with the study of the Galactic lithium evolution with metallicity is that lithium is depleted in stellar interiors along the life of the star, and some Li production can even occur at specific evolved stages and cannot be assumed to be representative of the Li abundance of the ISM material from which the star was formed. This phenomenon leads to a broad spread in $A_{\mathrm{Li}}$ whatever the metallicity. The metallicity in our catalogue covers a broad range, from $[\mathrm{M} / \mathrm{H}]=-3$ dex for halo stars to $[\mathrm{M} / \mathrm{H}]=+0.5 \mathrm{dex}$ for the richest disc stars. The goal of the present section is to investigate the ISM lithium abundance variation along this wide metallicity range. To accomplish this, we selected hot stars from the working sample with $T_{\text {eff }}>5600 \mathrm{~K}$ to reject the coolest dwarf stars with deeper convective zone (higher lithium destruction), thereby creating a clean sample of 2310 stars.

\subsubsection{The AMBRE stars of the Spite plateau}

For more than three decades hot metal-poor stars have been known to exhibit a rather constant lithium abundance with metallicity $\left(A_{\mathrm{Li}} \sim 2.2\right.$ dex; Spite \& Spite 1982). This plateau was first interpreted as the primordial lithium abundance, but since the recent results of the Wilkinson Microwave Anisotropy Probe (WMAP) mission, the lithium abundance of the Standard Big Bang Nucleosynthesis (SBBN) has been revised to $A_{\mathrm{Li}} \sim 2.6 \mathrm{dex}$ (Spergel et al. 2003). This discrepancy is know as the lithium problem, and several studies proposed different depletion mechanisms to explain such a difference, (e.g. Pinsonneault et al. 1999).

We present the NLTE lithium abundances of the 44 metalpoor stars $([\mathrm{M} / \mathrm{H}]<-1.5 \mathrm{dex})$ of the clean sample in Fig. 8. 

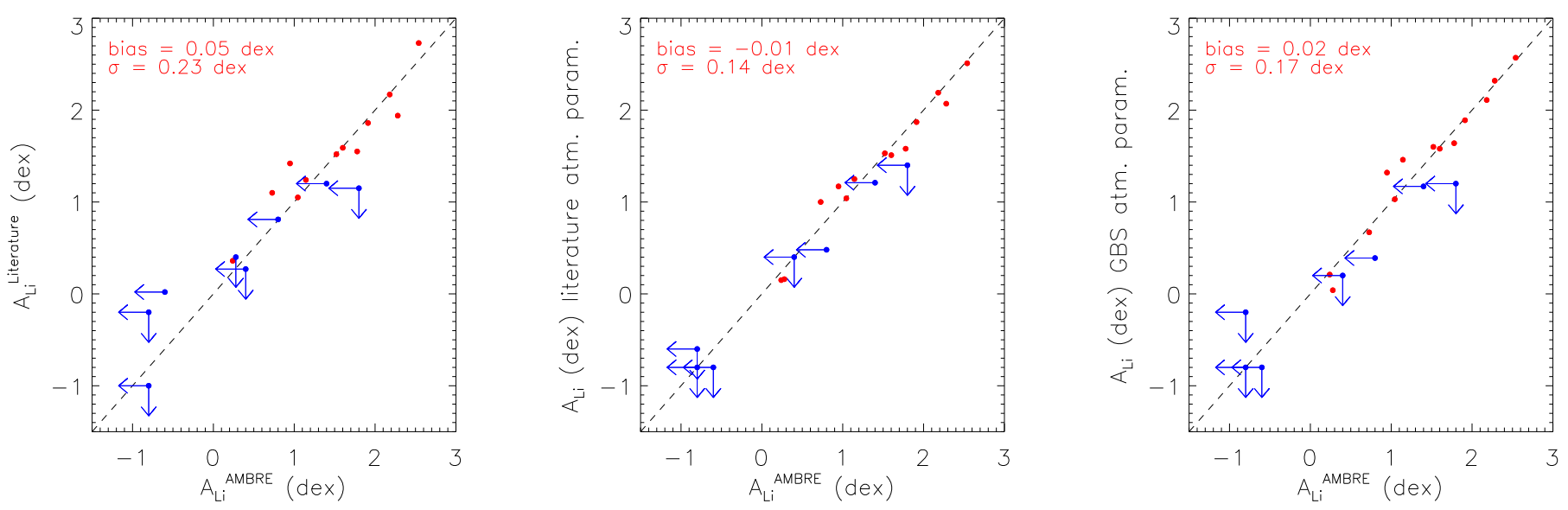

Fig. 7. LTE lithium abundances for a set of 20 Gaia-benchmark stars. Left panel: literature abundances compared to the AMBRE/Li determinations. Middle panel: lithium abundances derived from the AMBRE spectra and adopting the literature atmospheric parameters compared to the AMBRE/Li determinations. Right panel: lithium abundances derived from the AMBRE spectra and adopting the Gaia benchmark stars (GBS) atmospheric parameters compared to the AMBRE/Li determinations. Lithium abundances are shown in red, while upper limits are represented by blue arrows. Mean differences and standard deviations for the lithium abundance are reported. Stars and references of the literature values are the following: $\epsilon$ Eri, $\beta$ Vir (Gonzalez et al. 2010); $\alpha$ Cen A, 18 Sco (Baumann et al. 2010); HD 22879 (Nissen \& Schuster 2012); Sun (Grevesse et al. 2007); $\tau$ Cet (Takeda $\&$ Kawanomoto 2005); $\alpha$ Cen B (Chmielewski et al. 1992); $\mu$ Ara, $\delta$ Eri, $\beta$ Hyi (Bruntt et al. 2010); Procyon, HD 49933 (Ramírez et al. 2012); HD 84937 (Lind et al. 2013); HD 140283 (Asplund et al. 2006); $\epsilon$ For (Lèbre et al. 1999); Arcturus (Reddy \& Lambert 2005); $\epsilon$ Vir, $\alpha$ Tau (Mallik 1999); and HD 107328 (Brown et al. 1989).

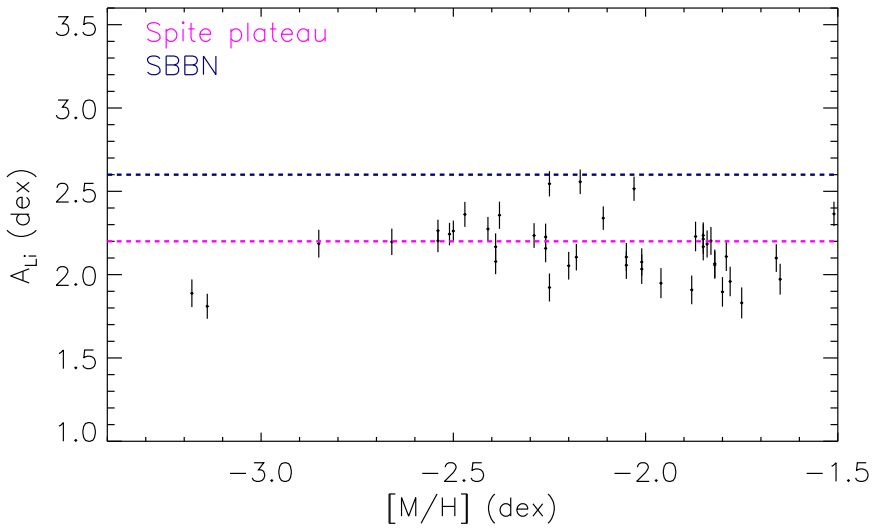

Fig. 8. $A_{\mathrm{Li}}^{\mathrm{NLTE}}$ of the AMBRE/Li catalogue stars as a function of $[\mathrm{M} / \mathrm{H}]$ for $[\mathrm{M} / \mathrm{H}]<-1.5$ dex. We overplotted the typical SBBN and Spite plateau lithium abundances in blue and magenta, respectively.

First, we clearly see that stars with $-3.0<[\mathrm{M} / \mathrm{H}]<-1.5 \mathrm{dex}$ show a rather constant lithium abundance $\left(\left\langle A_{\mathrm{Li}}\right\rangle=2.08 \mathrm{dex}\right)$ with a typical dispersion of 0.22 dex. We thus confirm that the mean lithium abundance of the most metal-poor stars in the Galaxy is lower by $\sim 0.4 / 0.5$ dex with respect to the SBBN value. Second, we found two stars with $[\mathrm{M} / \mathrm{H}]<-3$ dex and typical lithium abundances that are much lower than the Spite plateau $\left(A_{\mathrm{Li}}^{\mathrm{AMBRE}}=1.81,1.88 \mathrm{dex}\right)$. These stars have already been analysed by Sbordone et al. (2010) with the same UVES data and our lithium abundances are consistent with these within $2 \sigma$ errors $\left(A_{\mathrm{Li}}^{\text {Sbordone }}=2.10,1.65 \mathrm{dex}\right)$. The differences between the spectroscopic AMBRE $T_{\text {eff }}$ and the photometric temperatures of Sbordone et al. (2010) are $-413 \mathrm{~K}$ and $+528 \mathrm{~K}$, respectively, and can explain such differences in abundances. A reason why these stars show lower lithium abundances compared to the Spite plateau could be that they suffered from a subsequent lithium depletion, as proposed by Sbordone et al. (2010). Also, we find three stars with lithium abundances that are higher that $A_{\mathrm{Li}}>2.5 \mathrm{dex}\left(A_{\mathrm{Li}}^{\mathrm{AMBRE}}=2.54,2.51,2.56 \mathrm{dex}\right)$. These targets were already observed by Lind et al. (2009b) and our lithium abundances are consistent with these targets within $2 \sigma$ errors $\left(A_{\mathrm{Li}}^{\mathrm{Lind}} 2.31,2.19,2.16 \mathrm{dex}\right)$. The differences between the AMBRE $T_{\text {eff }}$ and the photometric $T_{\text {eff }}$ of Lind et al. (2009b) are $+567 \mathrm{~K},+361 \mathrm{~K}$, and $+407 \mathrm{~K}$, respectively, and can again explain such differences in abundances. Lind et al. (2009b) used GIRAFFE/HR15 data $(R \sim 19200)$ contrary to UVES data in our study.

\subsubsection{ISM lithium abundance vs. $[\mathrm{M} / \mathrm{H}]$}

As emphasized previously, lithium depletion mechanisms seem to occur at all metallicities, especially for $[\mathrm{M} / \mathrm{H}]>-1.5 \mathrm{dex}$. To study the lithium abundance in the ISM, one has to consider the upper envelope of the lithium distribution that reflects the initial condition of the ISM to a greater extent, i.e. stars with no lithium depletion. For that purpose, we followed the same approach as Lambert \& Reddy (2004) and Delgado Mena et al. (2015). We regularly binned the data of the clean sample with a step $\Delta[\mathrm{M} / \mathrm{H}]=+0.10$ dex for the range $-1 \leq[\mathrm{M} / \mathrm{H}] \leq$ +0.5 dex to trace $A_{\mathrm{Li}}$ as a function of the metallicity. We also considered two bins with $\Delta[\mathrm{M} / \mathrm{H}]=+0.20$ dex on the domain $-1.4<[\mathrm{M} / \mathrm{H}]<-1.0 \mathrm{dex}$. This final subsample consists of a total of 2264 stars. Errors on lithium abundances are typically 0.1 dex. For all the metallicity bins, we then chose the six stars with the highest NLTE abundance in lithium and computed their mean $A_{\mathrm{Li}}$. We adopted this amount of stars per bin as in previous studies, but we confirm that our results are robust when selecting 4, 6 , or 8 stars. For a given bin, the error bar is finally given by the standard deviation of the $6 A_{\mathrm{Li}}$ measurements. The AMBRE relation shown in Fig. 9 is stable with both the metallicity binning and the cut in $T_{\text {eff }}$ and $\log (g)$. We also emphasize the fact that it is the first time that this type of study is carried out with such a large statistical sample size and a perfectly homogeneous catalogue over a very wide metallicity range.

It can be seen in Fig. 9 that for $[\mathrm{M} / \mathrm{H}]<-1$ dex, the AMBRE relation is consistent with the Spite plateau, as with the point of Lambert \& Reddy (2004). However, the three points from Delgado Mena et al. (2015) show a strong disagreement 


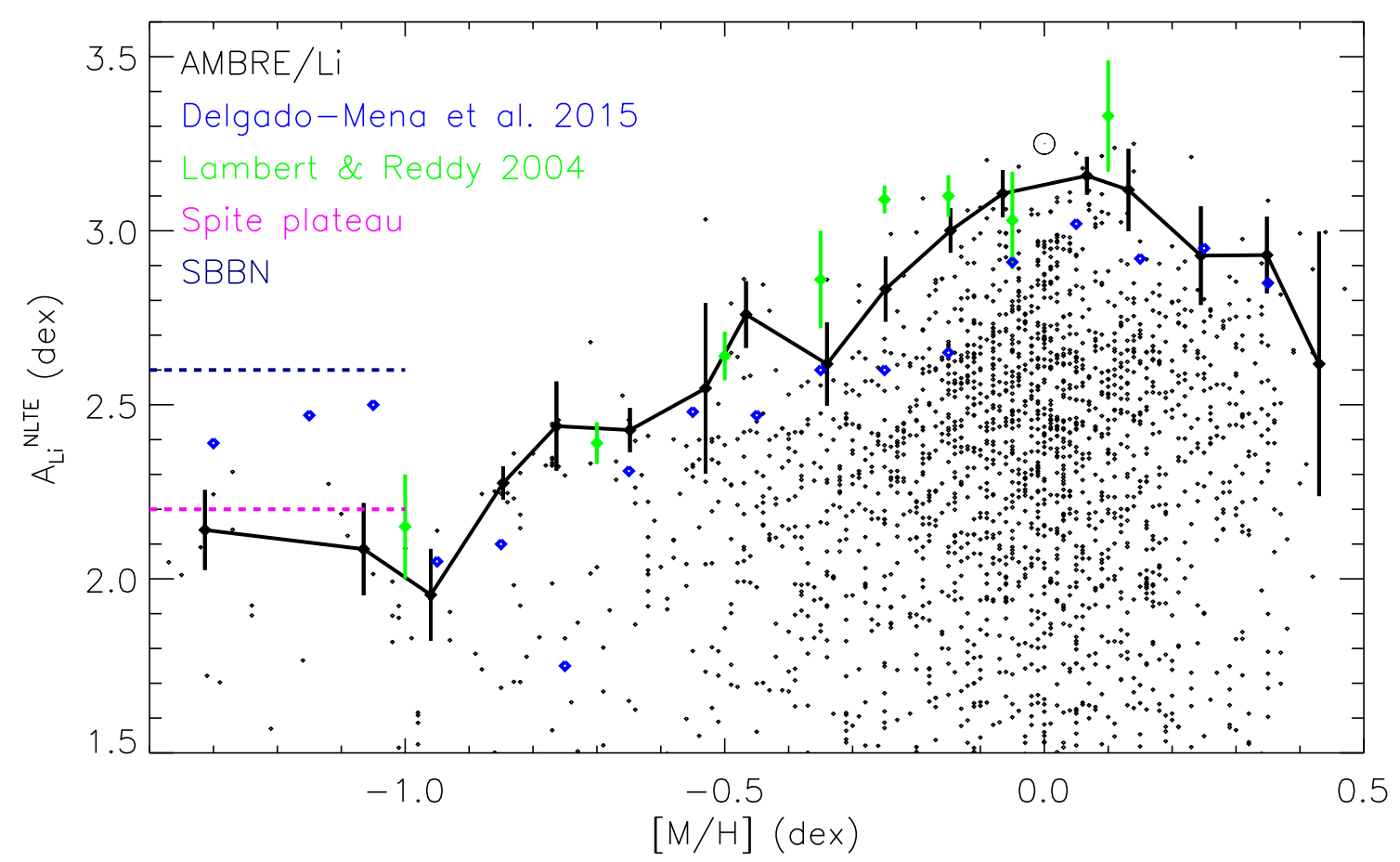

Fig. 9. Mean lithium value for the 6 stars with the highest lithium abundance in each metallicity bin as a function of the metallicity. The AMBRE relation is shown in black, while the relation of Lambert \& Reddy (2004) and Delgado Mena et al. (2015) are overplotted in green and blue, respectively. We overplotted in black all the AMBRE individual lithium measurements used to build the black curve together with the solar meteoritic lithium abundance.

with our data, probably because their last two bins are composed of only one star (see their Fig. 5). Moreover, we clearly measure an increase of the maximum lithium abundance in the range $-1.0 \lesssim[\mathrm{M} / \mathrm{H}] \lesssim+0.1$ dex, reaching $A_{\mathrm{Li}}=3.16 \mathrm{dex}$ in the domain $+0.0 \leq[\mathrm{M} / \mathrm{H}] \leq+0.1$ dex, which is close to the meteoritic abundance value. It can be seen that our AMBRE curve is in a rather good agreement with Lambert \& Reddy (2004), while the relation of Delgado Mena et al. (2015) is systematically lower, possibly due to sample selection effects. At super-solar metallicity, the maximum lithium abundance decreases clearly by a factor 0.55 dex in the range $+0.00<[\mathrm{M} / \mathrm{H}]<+0.50$ dex. We are confident with this behaviour since it is constrained by five data points.

For $[\mathrm{M} / \mathrm{H}]>+0.0$ dex, we visually checked that the quality of the fit between the observed and synthetic spectrum is good for the six stars with the highest lithium abundance in each metallicity bin. The average $T_{\text {eff }}$ of these six richest stars tends to increase with the metallicity, so that in this situation internal destruction of lithium does not seem to be the reason for the decrease. In addition, the errors in $T_{\text {eff }}$ and $A_{\mathrm{Li}}$ tend to decrease with the metallicity, while for $[\mathrm{M} / \mathrm{H}]$ the average error is very constant.

Several Galactic chemical evolution models (GCE) tried to reproduce the rise of lithium with metallicity by considering the contributions of several sources: spallation by GCC, the $v$ process by the core-collapse supernovae (CCSN), novae, lowmass giants and AGB stars, starting from a primordial lithium value $\left(\mathrm{SBBN}, A_{\mathrm{Li}}=2.6\right.$ dex). Fields \& Olive (1999) developed a GCE, considering only the GCC and CCSN contributions; the model cannot fit the meteoritic lithium abundance because the author did not include stellar sources. Later, Romano et al. (2001) proposed a model with the five previously quoted sources and a dominant low-mass star contribution that fits the meteoritic value rather well. The same year Travaglio et al. (2001) concluded that the major contribution comes from AGBs, while the role of low-mass giants, novae, and CCSNs is weak. Carbonrich AGB stars were proposed by Alibés et al. (2002) as the main source of lithium at $[\mathrm{M} / \mathrm{H}]>-0.5$ dex.

More recently, Prantzos (2012) provided a new GCE model for the light elements $\mathrm{Li}, \mathrm{Be}$, and $\mathrm{B}$, considering all possible sources of lithium: SBBN, AGBs, low-mass giants, novae, GCRs, and CCSN. He found that i) the two best-known sources of $\mathrm{Li}$, namely SBBN and GCR, can provide about $10 \%$ and $20 \%$ of the solar $\mathrm{Li}$, respectively, leaving the remaining $70 \%$ for a stellar source; and ii) current yields from all the aforementioned stellar sources fail to provide the remaining amount by factors $\sim 5-10$, thus calling for a considerable reassessment of Li production in stars. He also argued that the uncertainties in stellar $\mathrm{Li}$ yields and the amount of Li depletion in stellar envelopes make it difficult to make any meaningful comparison between GCE models and Li observations.

The behaviour of $A_{\mathrm{Li}}$ at supersolar metallicities reported here, namely its decrease for $[\mathrm{M} / \mathrm{H}]>+0.0 \mathrm{dex}$, is not reproduced by the aforementioned models with the possible exception of Fields \& Olive (1999). However, that model neglected the main stellar sources of $\mathrm{Li}$, thus failing to reproduce the solar $\mathrm{Li}$ value, and the reasons for the obtained decrease is not clear. The Li decrease revealed by the AMBRE/Li data analysis is unique (no other element, besides D, displays a decreasing abundance) and requires further investigation on theoretical grounds and an independent observational confirmation.

\subsection{Lithium in the thin and thick discs}

It is now well established that the Milky Way disc has two major components. Indeed, the presence of a thin and thick disc has been revealed by many methods, for example by stellar counts (Gilmore \& Reid 1983), dynamically (Bensby et al. 2014) and 
chemically by $[\alpha / \mathrm{Fe}]$ ratio abundances (e.g. Adibekyan et al 2011; Recio-Blanco et al. 2014), suggesting at least two different evolutionary paths in the history of the Milky Way disc formation.

In this context, two recent works attempted to study both discs in order to detect a possible distinct chemical lithium evolution in the thin and thick discs. Ramírez et al. (2012) observed that the thin disc stars show a rather high lithium content, marked by an enrichment in $A_{\mathrm{Li}}$ with increasing $[\mathrm{M} / \mathrm{H}]$, while the maximum thick disc lithium abundances are constant with $[\mathrm{M} / \mathrm{H}]$ and very close to the Spite plateau. By decomposing the thin disc in age slices ${ }^{3}$, Ramírez et al. (2012) also showed that the lithium abundance envelope of the thin disc is in continuity with that observed in the thick disc. These authors applied a kinematical criteria to separate thin and thick stars. In a more recent work, Delgado Mena et al. (2015) proposed that the thick disc lithium abundances decrease with increasing metallicity in contrast with Ramírez et al. (2012), while the thin disc shows higher lithium content than the thick disc (as already revealed by Ramírez et al. 2012). Delgado Mena et al. (2015) disentangled both discs thanks to the kinematical criteria of Ramírez et al. (2012), as well as by searching for gaps in the $[\alpha / \mathrm{Fe}]$ distribution for a given metallicity range. Delgado Mena et al. (2015) concluded that these two thin to thick disc separations do not influence their results.

We used the AMBRE/Li catalogue to search for a possible distinction in lithium between both Galactic discs. The high statistics and homogeneity of this catalogue offer a good opportunity to perform such an analysis. In contrast to the two previous quoted studies, we are able to provide a new view on this problem. In order to chemically separate thin to thick disc stars, we built a clean sample from the working sample presented at the beginning of this section, composed of stars with the best atmospheric parameters, i.e. QUALITY_FLAG $=0$ and $S / N>150$ to establish a robust chemical separation. We also rejected stars with $[\mathrm{M} / \mathrm{H}] \leq-1.25$ dex that are probably halo members and weakly representative of the thin and hick discs in terms of metallicity. The resulting clean sample is composed of 363 very well parametrized stars. It is used to define our methodology for the thin/thick disc classification.

We used the $[\alpha / \mathrm{Fe}]$ ratio and $[\mathrm{M} / \mathrm{H}]$ provided by the AMBRE project to disentangle the two discs. We followed the same procedure as in Guiglion et al. (2015). We decomposed the $[\mathrm{M} / \mathrm{H}]$ distribution of our clean sample in metallicity bins, searching visually for gaps in the corresponding $[\alpha / \mathrm{Fe}]$ distribution. We were able to separate two components between $-1.1 \leq[\mathrm{M} / \mathrm{H}] \leq-0.15$ dex. We linearly fitted these gaps to separate high- $[\alpha / \mathrm{Fe}]$ stars (thick disc with $-1.25 \leq[\mathrm{M} / \mathrm{H}] \leq$ -0.15 dex) from low- $[\alpha / \mathrm{Fe}]$ stars (thin disc with $-1.25 \leq$ $[\mathrm{M} / \mathrm{H}] \leq-0.50 \mathrm{dex})$, following an extrapolated separation on the domain $-1.25 \leq[\mathrm{M} / \mathrm{H}] \leq+0.50$, as shown in Fig. 10. Metalrich $\alpha$-rich stars with $[\mathrm{M} / \mathrm{H}]>-0.15$ dex and $[\alpha / \mathrm{Fe}]$ above the separation were rejected because they are too metal-rich compared to the classical definition of the thick disc. We finally applied this separation to the whole working sample on the same metallicity domain (3009 stars, 89\% thin disc, 7\% thick disc, and $4 \%$ metal-rich $\alpha$-rich stars). The thick disc stars are in the minority compared to the thin disc stars; this is probably because the AMBRE content of the ESO archive is not optimized to target thick disc stars as for example in the Gaia-ESO Survey.

\footnotetext{
3 Ramírez et al. (2012) determined the ages thanks to an isochrone fitting method and distances from Hipparcos parallaxes.
}

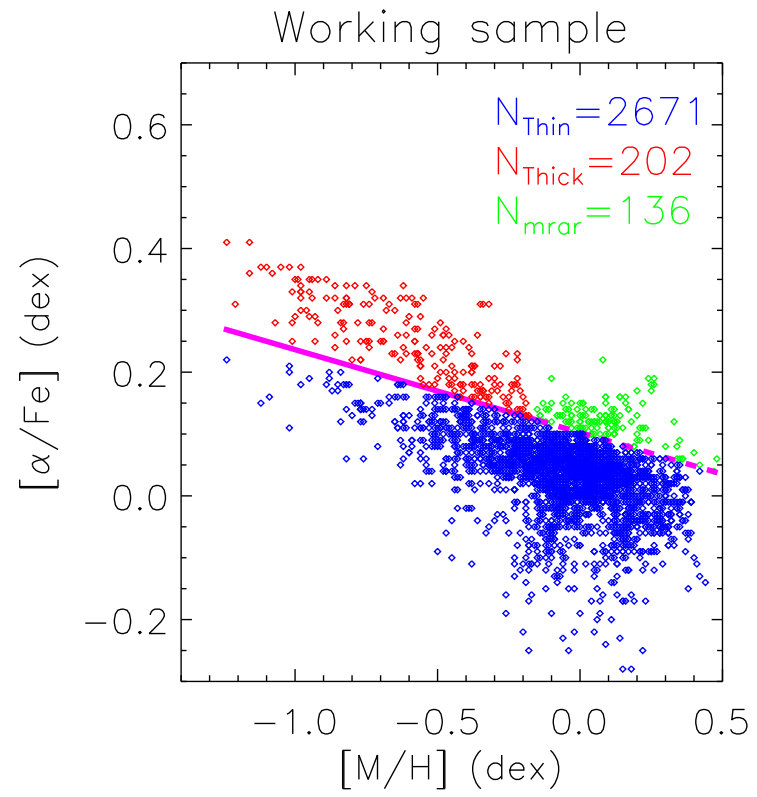

Fig. 10. $[\alpha / \mathrm{Fe}]$ ratio as a function of $[\mathrm{M} / \mathrm{H}]$ of the AMBRE/Li stars for the working sample ( 3009 stars). The full magenta line shows the thin to thick disc separation. The magenta dashed line shows the extrapolated separation for $[\mathrm{M} / \mathrm{H}]>-0.15 \mathrm{dex}$. The thin disc stars are colour-coded in blue, while thick disc members are in red. The metal-rich $\alpha$-rich stars are shown in green.

Based on this thin to thick disc characterization, the lithium content in each disc is shown in Fig. 11, for the working sample.

- The AMBRE thick disc stars exhibit lower abundances of lithium than those in the thin disc and the highest lithium abundances seem to increase slightly with $[\mathrm{M} / \mathrm{H}]$, around $A_{\mathrm{Li}} \sim 2-2.2$ dex. In addition, the normalized distribution of $A_{\mathrm{Li}}$ in the thick disc clearly shows two peaks at $\sim 2.2$ and $\sim 1.2$ dex, respectively. The higher value seems to correspond to an extension of the Spite plateau, while the lower value is certainly due to internal lithium destruction. Our results for the thick disc are slightly different from the study of Ramírez et al. (2012) described earlier and contradict the decrease of lithium with $[\mathrm{M} / \mathrm{H}]$ in the thick disc shown by Delgado Mena et al. (2015).

- As shown in Fig. 11, the lithium abundance for the thin disc stars increases with metallicity, reaching the highest values around solar metallicity and decreases at super-solar metallicity (as already noted in Sect. 6.1). The normalized $A_{\mathrm{Li}}$ distribution of the thin disc is characterized by a single peak $\left(A_{\mathrm{Li}} \sim 2.5 \mathrm{dex}\right)$ with a tail towards lower $A_{\mathrm{Li}}$. The rise of the upper lithium envelope of the thin disc is in agreement with Ramírez et al. (2012) (as shown in the Fig. 11), while we confirm the decrease of $A_{\mathrm{Li}}$ at super-solar metallicity, suggested in data from Delgado Mena et al. (2015).

We also observe that the lithium abundance distribution of the metal-rich $\alpha$-rich stars has a peak at $A_{\mathrm{Li}} \sim 3 \mathrm{dex}$, with a tail extending to lower lithium abundances. The higher peak compared to the thin and thick disc could indicate a different ISM enrichment history. In addition, a link with the Galactic bulge is not obvious because of numerous stars with $A_{\mathrm{Li}}>2.5 \mathrm{dex}$, compared to bulge studies pointing out stars with $A_{\mathrm{Li}}<2.5 \mathrm{dex}$ (Pompéia et al. 2002; Barbuy et al. 2010).

Our study is based on a thin to thick disc separation using a chemical criterion in contrast to Ramírez et al. (2012) 

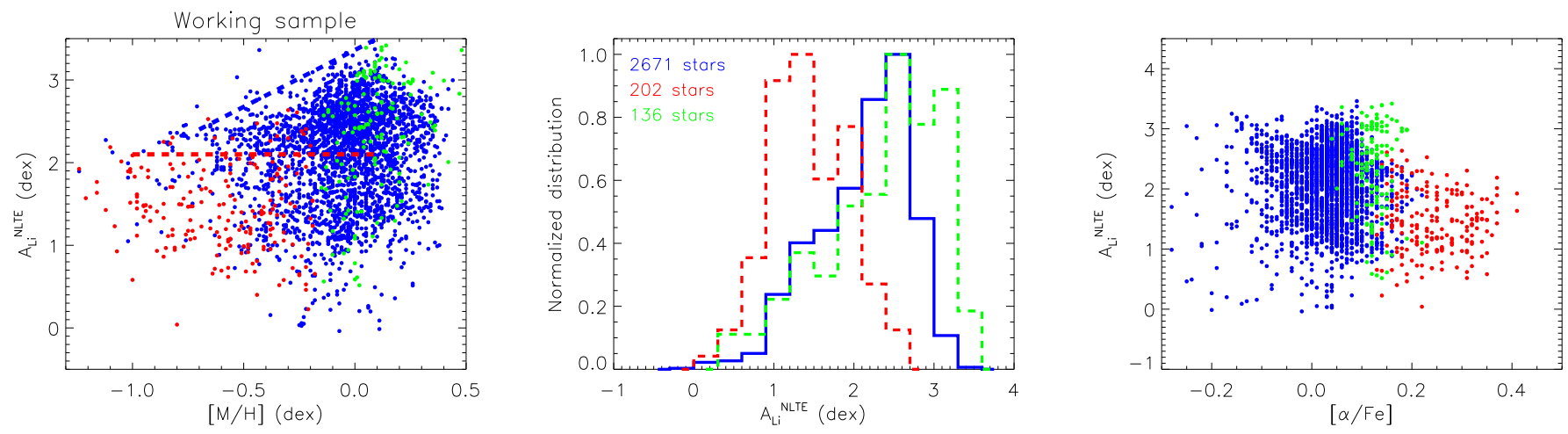

Fig. 11. $A_{\mathrm{Li}}^{\mathrm{NLTE}}$ as a function of $[\mathrm{M} / \mathrm{H}]$ (left) and its normalized distribution (centre) of the AMBRE/LI stars for the working sample. The thin disc stars are plotted in blue, thick disc members in red and metal-rich $\alpha$-rich stars in green. The upper envelope of both discs from Ramírez et al. (2012) are shown as dashed lines (bottom, left). We also show $A_{\mathrm{Li}}^{\mathrm{NLTE}}$ vs. $[\alpha / \mathrm{Fe}]$ in the right panel (Spearman's rank correlation coefficient equal to -0.03 and -0.04 for the thin and the thick disc, respectively).

who applied a dynamical selection. Moreover, as suggested by Ramírez et al. (2012), we emphasize the fact that these results can suffer from biases in ages, masses, and metallicity between both discs, and then show different degrees of lithium depletion or atomic diffusion, instead of real lithium enrichment. However, the agreement is real between both studies.

In Fig. 11 (right panel), it can be suggested that in the thick disc, the upper envelope of the stellar lithium content is correlated with the $[\alpha / \mathrm{Fe}]$. As the $[\alpha / \mathrm{Fe}]$ ratio is well correlated with the age in the thick disc (see for example Haywood et al. 2013), we directly see the evolution of lithium with time. On the other hand, no clear trend of $A_{\mathrm{Li}}$ is observed in the thin disc with $[\alpha / \mathrm{Fe}]$.

From the Galactic chemical evolution point of view, our finding confirms that the ISM where the thick disc stars were formed has not been significantly enriched from the Spite plateau abundance value $\left(A_{\mathrm{Li}} \sim 2.2 \mathrm{dex}\right)$. In the model of Prantzos (2012, see e.g. Fig. 16 of that work), the ISM has been weakly enriched by CCSNs and GCRs at low metallicity $([\mathrm{M} / \mathrm{H}]<-0.6 \mathrm{dex})$, whereas low-mass stars are able to enrich sensitively the ISM only at higher metallicity $([\mathrm{M} / \mathrm{H}]>-0.6 \mathrm{dex})$, corresponding to the thin disc phase. However, the observed double-branch behaviour of $\mathrm{Li}$ cannot be interpreted in the framework of the simple 1-zone model of Prantzos (2012) or other previous GCE models. For the moment, we can only infer that the lowmass stellar sources of Li have only played a key role in the ISM enrichment of the thin disc.

Finally, we repeated the analysis presented in Sect. 6.1 to investigate the lithium content of the ISM in which the thin and thick disc were formed. We removed the stars with $T_{\text {eff }}<5600 \mathrm{~K}$ as in Sect. 6.1, leading to fewer stars compared to Fig. 11. We show our results in Fig. 12. While the thick disc shows a slightly increasing maximum abundance in spite of the cut in $T_{\text {eff }}$, the thin disc shows a typical increase of lithium abundance with $[\mathrm{M} / \mathrm{H}]$ to lithium meteoritic abundance at solar metallicity. The important highlight is that we are able to show the lithium decrease at super-solar metallicities in the thin disc. The two distinct relations in the thin and thick disc indicate then that both discs are characterized by two distinct lithium enrichments.

Recent chemical evolution models manage to produce a "thick disc" (older than $\sim 8-9$ Gyr) through radial migration of stars from the inner disc. This leads naturally to two branches in the $[\mathrm{O} / \mathrm{Fe}]-[\mathrm{Fe} / \mathrm{H}]$ space corresponding to the observations of the thin and the thick disc (e.g. Schönrich \& Binney 2009; Minchev et al. 2013; Kubryk et al. 2015). Work is now in progress to study the lithium evolution of the thin and thick

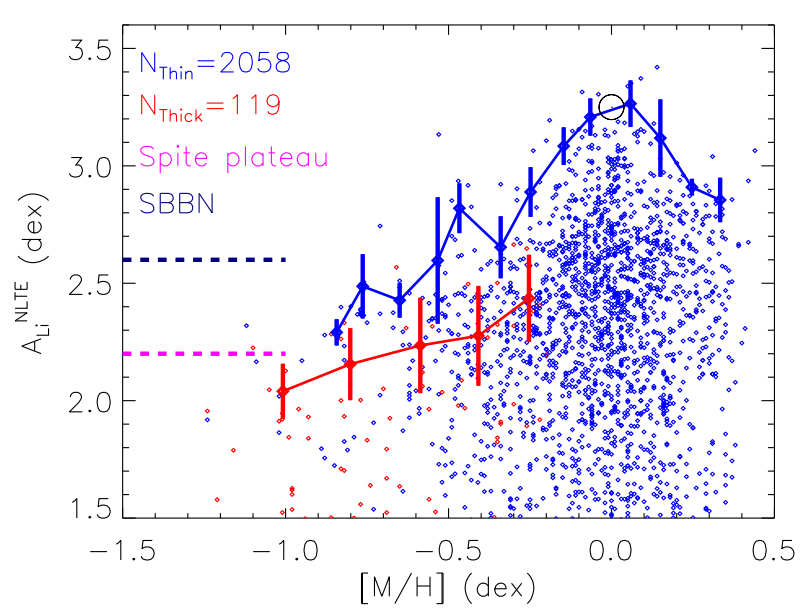

Fig. 12. Same figure as Fig. 9, but taking into account the thin (in blue) to thick (in red) characterization.

discs in the framework of the model of Kubryk et al. (2015), but lithium destruction and atomic diffusion in stellar interiors makes the situation more complex than for oxygen (Prantzos et al., in prep.).

\section{Summary}

Produced during the Big Bang, in stars, and in the ISM, lithium can also be easily burnt in stellar interiors. This chemical species therefore shows a great interest when one tries to understand its chemical evolution in our Galaxy, the Milky Way.

In order to understand its chemical evolution history, we built a homogeneous catalogue of lithium abundance composed of 7300 stars and based on high-resolution FEROS, HARPS, and UVES spectra parametrized within the AMBRE project. We performed a fast automatic determination of lithium abundances by coupling a synthetic spectra grid and the Gauss-Newton algorithm GAUGUIN. It is the first time that such a massive catalogue is built in a very homogeneous way compared to previous studies based on few hundreds of stars.

The AMBRE/Li catalogue covers a large domain in metallicity and evolutionary stages. These derived lithium abundances were validated by comparison with independent and recent literature values based on HARPS spectra. An additional check for the Gaia benchmark stars was also performed. From this large and unique data set, we studied the lithium content in the Milky Way together with its temporal evolution. 
First, based on a subsample of 2310 dwarf stars, we showed that the ISM lithium abundance increases by $\sim 1$ dex over the domain $-1.0 \leq[\mathrm{M} / \mathrm{H}] \leq+0.0 \mathrm{dex}$. This result is qualitatively in agreement with chemical evolution models of lithium in the Milky Way (Romano et al. 2001; Prantzos 2012). We also find a singular behaviour for lithium, whose ISM abundance decreases by about 0.5 dex at super-solar metallicities $(+0.0 \leq[\mathrm{M} / \mathrm{H}] \leq$ +0.5 dex). This behaviour is not predicted by current models.

Using a robust chemical separation, based on the enrichment on $\alpha$ elements with respect to iron, in our sample we identified stars as members of the thin and thick discs or members of a group of metal-rich $\alpha$-rich stars. We found that the thick disc stars suffered a low chemical enrichment, showing lithium abundances that are rather close to the Spite plateau. Nevertheless, the close thin disc stars clearly show a strong increase of their lithium content with metallicity, probably due to the contribution of low-mass stars. We concluded that the thin and thick discs clearly show two distinct lithium enrichment histories.

Acknowledgements. The spectra calculations were performed with the highperformance computing facility MESOCENTRE, hosted by OCA. C.C.W. would like to acknowledge the European Union FP7 programme through ERC grant number 320360 and the Leverhulme Trust through grant RPG-2012-541. This work was partly supported by the European Union FP7 programme through ERC grant number 32036. This work has made use of the VALD database, operated at Uppsala University, the Institute of Astronomy RAS in Moscow, and the University of Vienna.

\section{References}

Abia, C., Pavlenko, Y., \& de Laverny, P. 1999, A\&A, 351, 273

Adibekyan, V. Z., Santos, N. C., Sousa, S. G., \& Israelian, G. 2011, A\&A, 535, L11

Alibés, A., Labay, J., \& Canal, R. 2002, ApJ, 571, 326

Arnould, M., \& Norgaard, H. 1975, A\&A, 42, 55

Asplund, M., Lambert, D. L., Nissen, P. E., Primas, F., \& Smith, V. V. 2006, ApJ, 644, 229

Barbuy, B., Trevisan, M., Gustafsson, B., et al. 2010, in Light Elements in the Universe, eds. C. Charbonnel, M. Tosi, F. Primas, \& C. Chiappini, IAU Symp., 268, 325

Baumann, P., Ramírez, I., Meléndez, J., Asplund, M., \& Lind, K. 2010, A\&A, 519, A87

Bensby, T., Feltzing, S., \& Oey, M. S. 2014, A\&A, 562, A71

Bijaoui, A., Recio-Blanco, A., de Laverny, P., \& Ordenovic, C. 2012, Stat. Met., 9,55

Brooke, J. S. A., Bernath, P. F., Schmidt, T. W., \& Bacskay, G. B. 2013, J. Quant. Spectr. Rad. Transf., 124, 11

Brown, J. A., Sneden, C., Lambert, D. L., \& Dutchover, Jr., E. 1989, ApJS, 71, 293

Bruntt, H., Bedding, T. R., Quirion, P.-O., et al. 2010, MNRAS, 405, 1907

Chmielewski, Y., Friel, E., Cayrel de Strobel, G., \& Bentolila, C. 1992, A\&A, 263, 219

de Laverny, P., Recio-Blanco, A., Worley, C. C., \& Plez, B. 2012, A\&A, 544, A126

de Laverny, P., Clastres, C., Recio-Blanco, A., \& Worley, C. 2013a, Communication in "400 years of stellar rotation; The AMBRE Project: A catalog of Rotational Velocities of FGKM-type stars"

de Laverny, P., Recio-Blanco, A., Worley, C. C., et al. 2013b, The Messenger, 153, 18

De Pascale, M., Worley, C. C., de Laverny, P., et al. 2014, A\&A, 570, A68

Delgado Mena, E., Israelian, G., González Hernández, J. I., et al. 2014, A\&A, 562, A92

Delgado Mena, E., Bertrán de Lis, S., Adibekyan, V. Z., et al. 2015, A\&A, 576, A69

Fields, B. D., \& Olive, K. A. 1999, ApJ, 516, 797

Ghezzi, L., Cunha, K., Smith, V. V., et al. 2009, ApJ, 698, 451

Gilmore, G., \& Reid, N. 1983, MNRAS, 202, 1025

Gonzalez, G., Carlson, M. K., \& Tobin, R. W. 2010, MNRAS, 403, 1368

Grevesse, N., Asplund, M., \& Sauval, A. J. 2007, Space Sci. Rev., 130, 105
Guiglion, G., Recio-Blanco, A., de Laverny, P., et al. 2015, A\&A, 583, A91 Gustafsson, B., Edvardsson, B., Eriksson, K., et al. 2008, A\&A, 486, 951

Hartmann, D., Myers, J., Woosley, S., Hoffman, R., \& Haxton, W. 1999, in LiBeB Cosmic Rays, and Related X- and Gamma-Rays, eds. R. Ramaty, E. Vangioni-Flam, M. Cassé, \& K. Olive, ASP Conf. Ser., 171, 235

Haywood, M., Di Matteo, P., Lehnert, M. D., Katz, D., \& Gómez, A. 2013, A\&A, 560, A109

Heger, A., Langer, N., \& Woosley, S. E. 2000, ApJ, 528, 368

Heiter, U., Jofré, P., Gustafsson, B., et al. 2015, A\&A, 582, A49

Hinkle, K., Wallace, L., Livingston, W., et al. 2003, in The Future of Cool-Star Astrophysics: 12th Cambridge Workshop on Cool Stars, Stellar Systems, and the Sun, eds. A. Brown, G. M. Harper, \& T. R. Ayres, 851

Jofré, P., Heiter, U., Blanco-Cuaresma, S., \& Soubiran, C. 2014a, in Astron. Soc. India Conf. Ser., 11, 159

Jofré, P., Heiter, U., Soubiran, C., et al. 2014b, A\&A, 564, A133

Kubryk, M., Prantzos, N., \& Athanassoula, E. 2015, A\&A, 580, A126

Kupka, F., Piskunov, N., Ryabchikova, T. A., Stempels, H. C., \& Weiss, W. W. 1999, A\&AS, 138, 119

Kupka, F. G., Ryabchikova, T. A., Piskunov, N. E., Stempels, H. C., \& Weiss, W. W. 2000, Balt. Astron., 9, 590

Kurucz, R. L. 1992, Rev. Mex. Astron. Astrofis., 23

Lambert, D. L., \& Reddy, B. E. 2004, MNRAS, 349, 757

Lèbre, A., de Laverny, P., de Medeiros, J. R., Charbonnel, C., \& da Silva, L. 1999, A\&A, 345, 936

Lind, K., Asplund, M., \& Barklem, P. S. 2009a, A\&A, 503, 541

Lind, K., Primas, F., Charbonnel, C., Grundahl, F., \& Asplund, M. 2009b, A\&A, 503,545

Lind, K., Melendez, J., Asplund, M., Collet, R., \& Magic, Z. 2013, A\&A, 554, A96

Liu, Y. J., Tan, K. F., Wang, L., et al. 2014, ApJ, 785, 94

Lodders, K., Palme, H., \& Gail, H.-P. 2009, Landolt Börnstein [arXiv:0901.1149]

Mac Kellar, A. 1940, PASP, 52, 407

Mallik, S. V. 1999, A\&A, 352, 495

Mandell, A. M., Ge, J., \& Murray, N. 2004, AJ, 127, 1147

Masseron, T., Plez, B., Van Eck, S., et al. 2014, A\&A, 571, A47

Minchev, I., Chiappini, C., \& Martig, M. 2013, A\&A, 558, A9

Müller, E. A., Peytremann, E., \& de La Reza, R. 1975, Sol. Phys., 41, 53

Neckel, H. 1999, Sol. Phys., 184, 421

Nissen, P. E., \& Schuster, W. J. 2012, A\&A, 543, A28

Pinsonneault, M. H., Walker, T. P., Steigman, G., \& Narayanan, V. K. 1999, ApJ, 527,180

Plez, B. 2012, Turbospectrum: Code for spectral synthesis [record ascl:1205.004]

Pompéia, L., Barbuy, B., Grenon, M., \& Castilho, B. V. 2002, ApJ, 570, 820

Prantzos, N. 2012, A\&A, 542, A67

Ram, R. S., Brooke, J. S. A., Bernath, P. F., Sneden, C., \& Lucatello, S. 2014, ApJS, 211, 5

Ramírez, I., \& Allende Prieto, C. 2011, ApJ, 743, 135

Ramírez, I., Fish, J. R., Lambert, D. L., \& Allende Prieto, C. 2012, ApJ, 756, 46 Recio-Blanco, A., de Laverny, P., Kordopatis, G., et al. 2014, A\&A, 567, A5

Reddy, B. E., \& Lambert, D. L. 2005, AJ, 129, 2831

Reeves, H. 1970, Nature, 226, 727

Romano, D., Matteucci, F., Ventura, P., \& D'Antona, F. 2001, A\&A, 374, 646

Sackmann, I.-J., \& Boothroyd, A. I. 1992, ApJ, 392, L71

Sackmann, I.-J., \& Boothroyd, A. I. 1999, ApJ, 510, 217

Sansonetti, C. J., Richou, B., Engleman, Jr., R., \& Radziemski, L. J. 1995, Phys. Rev. A, 52, 2682

Sbordone, L., Bonifacio, P., Caffau, E., et al. 2010, A\&A, 522, A26

Schönrich, R., \& Binney, J. 2009, MNRAS, 396, 203

Sneden, C. A. 1973, Ph.D. Thesis

Sneden, C., Lucatello, S., Ram, R. S., Brooke, J. S. A., \& Bernath, P. 2014, ApJS, 214,26

Spergel, D. N., Verde, L., Peiris, H. V., et al. 2003, ApJS, 148, 175

Spite, F., \& Spite, M. 1982, A\&A, 115, 357

Tajitsu, A., Sadakane, K., Naito, H., Arai, A., \& Aoki, W. 2015, Nature, 518, 381

Takeda, Y., \& Kawanomoto, S. 2005, PASJ, 57, 45

Travaglio, C., Randich, S., Galli, D., et al. 2001, ApJ, 559, 909

Wallerstein, G., \& Sneden, C. 1982, ApJ, 255, 577

Woosley, S. E., Hartmann, D. H., Hoffman, R. D., \& Haxton, W. C. 1990, ApJ, 356,272

Worley, C. C., de Laverny, P., Recio-Blanco, A., et al. 2012, A\&A, 542, A48

Worley, C. C., de Laverny, P., Recio-Blanco, A., Hill, V., \& Bijaoui, A. 2016, A\&A, 591, A81

Yan, Z.-C., Tambasco, M., \& Drake, G. W. F. 1998, Phys. Rev. A, 57, 1652 